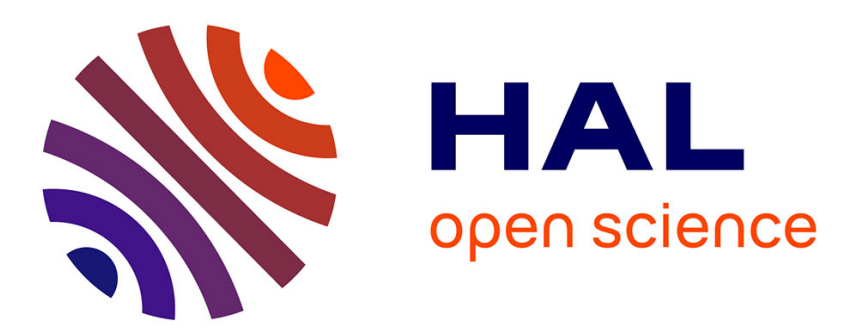

\title{
Relaxation dynamics in supercooled oligomer liquids: From shear-stress fluctuations to shear modulus and structural correlations
}

\author{
L. Klochko, J. Baschnagel, J. Wittmer, Alexander N Semenov
}

\section{- To cite this version:}

L. Klochko, J. Baschnagel, J. Wittmer, Alexander N Semenov. Relaxation dynamics in supercooled oligomer liquids: From shear-stress fluctuations to shear modulus and structural correlations. Journal of Chemical Physics, 2019, 151 (5), pp.054504. 10.1063/1.5110997 . hal-03560963

\section{HAL Id: hal-03560963 https://hal.science/hal-03560963}

Submitted on 7 Feb 2022

HAL is a multi-disciplinary open access archive for the deposit and dissemination of scientific research documents, whether they are published or not. The documents may come from teaching and research institutions in France or abroad, or from public or private research centers.
L'archive ouverte pluridisciplinaire HAL, est destinée au dépôt et à la diffusion de documents scientifiques de niveau recherche, publiés ou non, émanant des établissements d'enseignement et de recherche français ou étrangers, des laboratoires publics ou privés. 


\section{Relaxation dynamics in supercooled oligomer liquids: from shear-stress fluctuations to shear modulus and structural correlations}

L.Klochko, J.Baschnagel, J.P.Wittmer, A.N.Semenov*1

Institut Charles Sadron, CNRS - UPR 22, Université de Strasbourg, 23 rue du Loess, BP 84047, 67034 Strasbourg Cedex 2, France

(Dated: 9 July 2019)

Static and dynamical properties of a model glass-forming oligomer liquid are analysed using molecular dynamics (MD) simulations. The temperature and system size effects are assessed for the affine shear modulus $\mu_{A}$, the quasi-static shear modulus $\mu_{s f}$ (obtained using the stress-fluctuation relation) and the shear relaxation modulus $G(t)$. It is found that while both $\mu_{A}$ and $\mu_{s f}$ are nearly independent of the system size, their variances show significant system size dependence, in particular, below the glass transition temperature $T_{g}$. It is also shown that the standard deviation of the shear modulus, $\delta \mu_{s f}(T)$, exhibits a pronounced peak at $T \approx T_{g}$ whose position is nearly independent of the system volume $V$. Moreover, the whole function $\delta \mu_{s f}(T)$ is nearly the same for different system sizes above the glass transition. We propose a theory which quantitatively predicts $\delta \mu_{s f}(T)$ at $T \gtrsim T_{g}$ and explains both its independence of $V$ and its peak near $T_{g}$. It is also established that below $T_{g}$ the variance of the affine modulus follows the standard power law, $\left(\delta \mu_{A}\right)^{2} \propto 1 / V$, while $\delta \mu_{s f}$ shows anomalously slow decrease with $V$ as $\left(\delta \mu_{s f}\right)^{2} \propto 1 / V^{\alpha}$ with $\alpha<1$. On this basis it is argued that the studied glass-forming systems must show long-range structural correlations in the amorphous state. 


\section{INTRODUCTION}

The shear elastic modulus $\mu$ of a material is an important property allowing to discriminate between its liquid state (where $\mu=0$ ) and the solid state with $\mu>0$. Vitrifying liquids cooled below the glass transition temperature $T_{g}$ form amorphous solids which show long-time elastic response to small strains. The glass transition can be conveniently characterized by recording the long-time (quasi-equilibrium) modulus $\mu$ as a function of $T: \mu(T)$ strongly increases as the system is cooled near $T_{g} \cdot{ }^{1-6,25-29}$ Simulation studies suggest that this increase is sharp, but continuous ${ }^{1-5}$. Recent studies ${ }^{5,6}$ also reveal that glass transition is accompanied by strong fluctuations of the shear modulus $\mu$ : its standard deviation $\delta \mu$ shows a striking peak near $T_{g}$.

The study reported herein is inspired by these results. It has two goals: first, we analyzed the system size effects on the behavior of shear moduli and their fluctuations (cf. sections V, VI); second, we sought to clarify the physical meaning of the peak of $\delta \mu(T)$ and developed a quantitative theory accounting for it (cf. section VI). The first goal was achieved by doing new simulations of a smaller system (similar to that studied before ${ }^{5,6}$ ) and by their theoretical analysis (cf. sections III, IV, V, VI). Based on the two achievements we arrive at a rather coherent picture showing how fluctuations of elastic moduli and other properties of the studied glass-forming systems depend on their volume and temperature (cf. section VII). In particular, we show that the obtained results point to significant long-range spatial correlations within the inherent structures of the studied glassy systems. The model and the simulation approach are introduced in the next section. The main results are summarized in the last section VIII.

\section{THE MODEL AND ITS STATIC PROPERTIES}

\section{A. Model}

We studied the same oligomer glass-forming model system as used in the earlier work ${ }^{5-8}$. The system is a 3-dimensional model glassformer consisting of $M$ oligomer chains, each of 4 spherical particles ('monomers') connected by bonds. All unconnected monomers interact with a Lennard-Jones $(\mathrm{LJ})$ potential $u_{L J}(r)$ which is truncated and shifted at $r=r_{c u t}=2.3\left(r_{c u t} \approx 2 r_{\text {min }}\right.$ is roughly twice the distance for the potential minimum) ${ }^{6}$, so that $u_{L J}(r)=0$ for $r \geq r_{c u t}$ and $u_{L J}(r)=4\left(r^{-12}-r^{-6}\right)+$ const for $r \leq r_{c u t}$ (LJ units are used throughout the paper). The bond potential is harmonic: $u_{b}(r)=\left(k_{\text {bond }} / 2\right)\left(r-l_{\text {bond }}\right)^{2}$; the constants $k_{\text {bond }}=1110$ and $l_{\text {bond }}=0.967$ 
are chosen such that the probability for bond crossing is virtually null (the bond length cannot exceed $r_{c u t}$ for the same reason).

\section{B. Simulation protocol, density and RDF}

We performed standard MD simulations using a velocity-Verlet scheme with time step 0.005 in a cubic box with periodic boundary conditions ${ }^{9}$. The temperature $T$ and pressure $P$ are imposed using the Nosé-Hoover-Andersen algorithm ${ }^{10}$. We always set $P=0$ and start with NPT equilibration at $T=0.6$ which is well above the glass transition ${ }^{5,6}$. A large number $m=100$ of independent configurations are thus generated in this liquid regime. All the configurations are then slowly cooled down to $T=0.05$ with rate $\Gamma=-\partial T / \partial t=10^{-5}$ (at $P=0$ ). The emerging configurations are saved at a number of working temperatures $(T=0.55,0.5, \ldots 0.05)$. For each $T$ and for each system from the $m$-ensemble we then did (i) NPT tempering for time interval $\Delta t=10^{5}$ at $P=0$; (ii) determination of the average volume ${ }^{42}$ and switching to the NVT ensemble; (iii) NVT tempering over $\Delta t$ again; (iv) production NVT run, again over $\Delta t=10^{5}$.

The system of $M=3072$ short linear chains of LJ beads, with 4 beads per chain, was studied in refs. 5 and 6. We performed additional simulations of this system along with a detailed study of a new oligomer system with $M=768$ chains, and systematically compared various properties of the two systems. The linear dimension of the simulation box, $L$, for the smaller system $(768 \times 4)$ varies from $L=14.25$ to $14.72 \mathrm{LJ}$ units in the $T$-range from $T=0.05$ to 0.55 ; the size the larger system $(3072 \times 4)$ is between $L=22.6$ and 23.4 .

The $T$-dependence of the mean specific volume (per monomer) is shown in Fig. 1. The two systems have nearly the same density (the smaller system being a little bit less dense at low temperatures). The standard dilatometric criterion gives the glass transition at $T_{g} \approx 0.38$ for both systems (cf. refs. 5 and 6; note that the dilatometric $T_{g}$ is a reference estimate: generally $T_{g}$ depends on the cooling rate, $T_{g}$ is lower for a longer relaxation time-scale).

The radial distribution function $(\mathrm{RDF}) g_{n b}(r)$ for non-bonded monomer pairs is shown in Fig. 2 for both systems at two temperatures (above and below $T_{g}$ ). One can observe that the RDFs for the two systems are almost identical (the difference is not visible). The main peak just weakly depends on $T$ : it is only slightly more pronounced well below $T_{g}$ (at $T=0.25$ ). In all the cases the obtained RDFs show no sign of crystallization being typical for liquids (and disordered amorphous solids).

Thus we established that both systems are characterized by virtually the same density and the 
same pair correlation functions (including $g_{n b}(r)$ and the standard Kirkwood RDF, $g(r)$ ). This fact means that all the related static properties like energy, entropy, pressure and the affine shear modulus (see the next section) must be also nearly identical for both system sizes.

\section{Affine shear modulus}

The affine moduli are defined via stress response after an infinitesimal canonical affine transformation of positions and velocities of all particles ${ }^{6,31}$. In the case of shear in the xy plane such transformation reads

$$
x \rightarrow x+\gamma y, v_{y} \rightarrow v_{y}-\gamma v_{x}
$$

where $x, y$ and $v_{x}, v_{y}$ are coordinates and velocity components of a particle. (Note that there is a misprint in the kinetic part of the transformation in refs. 30 and 31: the subscripts $x$ and $y$ are interchanged there.) The instantaneous shear stress increment, $\delta \sigma_{x y}$, in the limit of small shear strain, $\gamma \rightarrow 0$, is linear in $\gamma$

$$
\left\langle\delta \sigma_{x y}\right\rangle \simeq \gamma \mu_{A}
$$

where the $\langle\ldots\rangle$ brackets signify an average over the statistical ensemble. The latter equation defines the ensemble-averaged shear modulus $\mu_{A}$ (which is also known as the affine modulus) ${ }^{5,6}$, and leads to the following general expression (cf. refs. 6 and 31): $\mu_{A}=\left\langle\tilde{\mu}_{A}\right\rangle$ where

$$
\tilde{\mu}_{A}=\frac{1}{V} \sum_{i} m_{i} v_{i x}^{2}+\frac{1}{V} \sum_{l} n_{y}^{2}\left[r^{2} u_{l}^{\prime \prime}(r) n_{x}^{2}+r u_{l}^{\prime}(r)\left(1-n_{x}^{2}\right)\right]
$$

Here 'tilde' indicates that the modulus is calculated for an instantaneous microstate of the system, $V$ is its volume, $m_{i}$ and $v_{i x}$ are the mass and $x$-velocity of the $i$-th particle. The first sum runs over all particles in the system, the second sum runs over all different pairs $(l)$ of interacting particles, $u_{l}(r)$ is the interaction potential for the $l$-pair, $u_{l}^{\prime}, u_{l}^{\prime \prime}$ are its first and second derivatives with respect to $r=|\underline{r}|$, where $\underline{r}=\underline{r}_{l}$ is the distance vector, $\underline{n}=\underline{r}_{l} / r_{l}$ is the pair orientation vector. The first term in the above equation is the kinetic (ideal-gas) contribution, while the second (excess) term is due to monomer interactions. In practice the kinetic term can be always ensemble averaged giving just $c T$, where $T$ is temperature in energy units $\left(T=k_{B} T_{a b s}\right.$ with $T_{a b s}$ being the absolute temperature), $c=N_{m} / V$ is monomer concentration, and $N_{m}=4 M$ is the total number of monomers in the system. While the chosen LJ potential is continuous, its derivative is not, giving rise to a singular contribution to $\mu_{A}$ known as an impulsive correction ${ }^{6,11}$ :

$$
\Delta \mu_{A}=-\frac{2 \pi}{15} c^{2} u_{L J}^{\prime}\left(r_{c u t}\right) r_{\text {cut }}^{4} g_{\text {nb }}\left(r_{\text {cut }}\right)
$$


The above equation is valid for 3-dimensional systems.

The obtained temperature dependencies of the time- and ensemble-averaged affine shear modulus for both system sizes are shown in Fig. 3a. (If not specified otherwise, an ensemble-averaging is taken over $m=100$ independent configurations and 3 shear planes, xy, yz and xz.) It is clear that $\mu_{A}$ is virtually the same for the two system sizes at all temperatures; it increases at low $T$ reflecting an increase of the system density.

\section{RELAXATION MODULUS AND STRESS CORRELATION FUNCTION}

The shear-stress relaxation modulus $G(t)$ is a central rheological characteristic of a liquid or solid material related to its dynamical moduli. ${ }^{12}$ This relaxation function can be obtained in a simple-shear experiment recording a shear-stress increment $\delta \sigma(t)$ generated by a small prescribed step-like shear deformation $\gamma_{0}$ at $t=0$ :

$$
G(t)=\lim _{\gamma_{0} \rightarrow 0}\langle\delta \sigma(t)\rangle / \gamma_{0}
$$

The instantaneous response is given by $G(0) \equiv \mu_{A}$. Note that a mathematically affine (that is, linear) deformation is assumed here. More precisely, it is a canonical affine transformation in the phase space that must be applied at $t=0.6,13$

The relaxation function $G(t)$ is closely related to the shear stress correlation function

$$
C(t)=\left\langle\sigma\left(t+t^{\prime}\right) \sigma\left(t^{\prime}\right)\right\rangle
$$

where $\sigma(t)=\sigma_{x y}(t)$ is the instant shear stress averaged over the system volume. As before $\langle\ldots\rangle$ means ensemble-averaging which is equivalent to averaging over $t^{\prime}$ for ergodic systems.

The stress correlation function $C(t)$ is connected to $G(t)$ via the stress-fluctuation equation $^{6,13,14}$ (relating the relaxation modulus in the linear response regime with fluctuations of the shear stress):

$$
G(t)=\frac{V}{T} C(t)+\text { const }
$$

where $V$ is the system volume and $T=k_{B} T_{a b s}$. Eq. 5 comes from the fluctuation-dissipation theorem (FDT) which is exact for equilibrium systems ${ }^{15,16}$. The last term (const) depends on the boundary conditions applied to obtain $C(t)^{3,5,6,17,31}$. For example const $=0$ with free boundary (in this case eq. 5 is exact only for $V \rightarrow \infty$ ) or when the boundary is coupled to a highly damping external medium ${ }^{17}$. The effect of such overdamped force was implemented in computations with 
periodic boundary conditions (PBC) using a hybrid MD-MC scheme ${ }^{17}$ involving canonical-affine shear deformations as MC moves. ${ }^{43}$ However, the constant in eq. 5 is generally nonzero in standard simulations with fixed PBC in the canonical ensemble.

While equation 5 is strictly valid for equilibrium systems (in particular, in the liquid state), it is also valid for glassy (supercooled) systems as argued below: In this latter case the system stays virtually trapped for a long time in a particular metabasin, MB (a group of inherent structures, IS, of the potential energy landscape in the configurational space ${ }^{18,19}$ ), so it becomes equilibrated within each MB. Hence, the FDT can be applied individually to each MB provided that transfer rates $f \sim 1 / \tau_{\alpha}$ between the glassy states (MBs) are very low, and therefore it must be valid also on the average (for the ensemble-averaged quantities) with any (generally, non-equilibrium) probability distribution between the glassy states (the MBs). The general condition for the FDT relation 5 to be valid is that the system must be equilibrated (prior to the measurements) during a long time strongly exceeding the time shift $t$ in $C(t)$. For $t \sim \Delta t$ the latter condition ensures that aging is negligible within the relevant time window $\Delta t$, as was verified earlier ${ }^{6}$.

Eq. 5 can be rewritten as ${ }^{6}$

$$
G(t)=\mu_{A}+\frac{V}{T}[C(t)-C(0)]
$$

The latter equation was employed to find the relaxation modulus for both systems at different temperatures. We obtained the correlation function $C(t)$ by averaging over $t^{\prime}$ (for the time interval $\sim \Delta t$ ), over the ensemble of $m=100$ independent systems, and over the 3 shear planes. The results are shown in Fig. 4 (the data for the larger system have been presented in refs. 5 and 6).

At short times, $t \lesssim 3, G(t)$ shows oscillations. These oscillations are not just a thermal noise, nor they are due to numerical errors: the oscillation pattern is reproducible and coincides for the two systems. It reflects vibrations of the molecular chain bonds ${ }^{20,21}$. It is, moreover, obvious that the whole $G(t)$ relaxation is independent of system size in the liquid regime $\left(T>T_{g}\right)$. Below $T_{g}$ the relaxation modulus shows a shoulder at $t \gtrsim 3$ which gets longer and gradually transforms into a plateau as $T$ is further decreased. It is remarkable that the $G(t)$ relaxation function is identical for both system at all temperatures $T>0.25$ (both above and below $T_{g}$ ) not only at short times, but also for a wider time-range, $t \lesssim 1000$, including most of the plateau regime. At longer times $\left(t \gtrsim 10^{4}\right)$ in the glassy regime $(T \lesssim 0.36)$ the relaxation of the smaller system $(768 \times 4)$ becomes retarded with respect to the larger one. For example, the apparent 'terminal' relaxation time is about twice longer for the smaller system at $T=0.25$.

What's the meaning of these results? The $G(t)$ relaxation at short times must reflect the iden- 
tical local structure of the two systems (in agreement with the RDF data). A slower long-time relaxation (longer $\tau_{\alpha}$ ) for smaller system below $T_{g}$ may seem counterintuitive (also in view of a slightly lower density of this system). Still this feature agrees with the previous simulation results and theoretical views on the glassy dynamics ${ }^{6,22,23}$ (in particular, it was shown ${ }^{22,23}$ that $\tau_{\alpha}$ for binary LJ mixtures increases as the system size decreases at $T$ below the onset of the glassy dynamics). This effect shows that the terminal $\alpha$-relaxation is not a local property but rather is a collective process involving transformations of large parts of the system. The corresponding dynamical correlation length (the size of the optimal cooperatively rearranging region) may thus exceed the size of the smaller system $(L \sim 14)$ leading to its slower relaxation (as the optimal relaxation pathway gets prohibited due to the system size). Such reasoning is also similar in spirit to the Adam-Gibbs-DiMarzio theory ${ }^{24}$ (cf. their argument based on the size dependence of the configurational entropy ${ }^{23}$ ). It is remarkable that at very low $T \lesssim 0.25$ the relaxation modulus $G(t)$ is a bit higher for the smaller system in the plateau regime. This effect is not due to a difference of the affine modulus $\mu_{A}=G(0)$, which is negligible between the two systems; rather it must be due to long-range relaxation modes which are effective for the larger system, but not for the smaller one.

Noteworthily, there is actually no contradiction between a lower density and a longer relaxation in the $768 \times 4$ system. The latter feature means that the $768 \times 4$ system must be a bit farther from equilibrium than the larger system at low $T$ in the glassy state. For density this means a stronger downward shift (cf. Fig. 1) from the equilibrium line, hence lower density as compared with the $3072 \times 4$ system.

\section{THE APPROACH TO OBTAIN THE SHEAR MODULUS AND ITS $T$-DEPENDENCE}

The long-time shear modulus can be defined simply as $\mu=G(\Delta t)$, where $\Delta t$ is the longest accessible laboratory (experimental or simulation) time-scale. Further, the function $G(t)$ can be obtained using the FDT relation, eq. 6, which takes the form

$$
G(t)=\mu_{A}-\mu_{0}+\frac{V}{T} C(t)
$$

with $\mu_{0}$ measuring the ensemble- and time-averaged square of the shear stress:

$$
\mu_{0}=\frac{V}{T} C(0)=\frac{V}{T}\left\langle\overline{\sigma^{2}}\right\rangle
$$


where

$$
\overline{\sigma^{2}}=\frac{1}{\Delta t} \int_{0}^{\Delta t} \sigma(t)^{2} \mathrm{~d} t
$$

The time-averaged stress is defined for each system of the ensemble as

$$
\bar{\sigma}=\frac{1}{\Delta t} \int_{0}^{\Delta t} \sigma(t) \mathrm{d} t
$$

As follows directly from the definition of $C(t)$

$$
\left\langle\bar{\sigma}^{2}\right\rangle=\frac{1}{\Delta t^{2}} \int_{0}^{\Delta t} C\left(t-t^{\prime}\right) \mathrm{d} t \mathrm{~d} t^{\prime}
$$

The above integral can be considered as an average of $C(t)$ over the time scale $\Delta t$. A similar average of $G(t)$ reads

$$
\mu_{s f} \equiv \frac{1}{\Delta t^{2}} \int_{0}^{\Delta t} G\left(\left|t-t^{\prime}\right|\right) \mathrm{d} t \mathrm{~d} t^{\prime}
$$

Obviously $\mu_{s f}$ depends on $\Delta t$. On using eqs. $7,10,11$ one gets ${ }^{5,6}$ :

$$
\mu_{s f}=\mu_{A}-\mu_{F}
$$

where

$$
\mu_{F}=\mu_{0}-\mu_{1}, \mu_{1}=\frac{V}{T}\left\langle\bar{\sigma}^{2}\right\rangle
$$

The modulus $\mu_{s f}$ defined in eq. 11 is dominated by the long-time stress response (for $t \sim \Delta t$ ), so $\mu_{s f}$ serves as a good and useful approximation for the terminal (quasi-static) modulus $\mu, \mu_{s f}(\Delta t) \approx$ $\mu(\Delta t)$, if the sampling time $\Delta t$ is long enough, $\Delta t \gg \tau_{0}$, where $\tau_{0}$ corresponds to the onset of the glassy elastic plateau below $T_{g}$ (see Fig. 4 where $\tau_{0} \sim 3$ ). Noteworthily, $\mu_{s f}(\Delta t)$ is better defined statistically than $\mu=G(\Delta t)$.

The fluctuation modulus $\mu_{F}$ measures the mean-square fluctuation of $\sigma$ over the sampling time $\Delta t:^{5,6}$

$$
\mu_{F}=\frac{V}{T}\left\langle\overline{(\sigma-\bar{\sigma})^{2}}\right\rangle
$$

On the other hand, it can be also considered as an effective drop of $G(t)$ during the time $\Delta t$ :

$$
\mu_{F}(\Delta t)=G(0)-\mu_{s f}(\Delta t)
$$

The modulus $\mu_{F}$ can be also treated as the average of individual moduli $\tilde{\mu}_{F}$ defined for each independent dynamical trajectory of a system from the ensemble:

$$
\mu_{F}=\left\langle\tilde{\mu}_{F}\right\rangle
$$


with

$$
\tilde{\mu}_{F}=\frac{V}{T}\left(\overline{\sigma^{2}}-\bar{\sigma}^{2}\right) \equiv \tilde{\mu}_{0}-\tilde{\mu}_{1}
$$

Apart from the factor $V / T, \tilde{\mu}_{0}$ is the mean-square shear stress of the trajectory, and $\tilde{\mu}_{1}$ is the square of the mean stress $\bar{\sigma}$. The latter equation can be rewritten as

$$
\tilde{\mu}_{F}=\frac{1}{2} \frac{V}{T}(\Delta t)^{-2} \int_{0}^{\Delta t}\left[\sigma\left(t_{1}\right)-\sigma\left(t_{2}\right)\right]^{2} \mathrm{~d} t_{1} \mathrm{~d} t_{2}
$$

Eq. 18 directly shows that any constant (quenched) stress does not affect $\mu_{F}$. Using eqs. 16, 18 we get:

$$
\mu_{F}(\Delta t)=\frac{2}{\Delta t^{2}} \int_{0}^{\Delta t}(\Delta t-t) h(t) \mathrm{d} t
$$

where

$$
h(t)=\frac{1}{2} \frac{V}{T}\left\langle\left[\sigma\left(t+t^{\prime}\right)-\sigma\left(t^{\prime}\right)\right]^{2}\right\rangle=\frac{V}{T}[C(0)-C(t)]=G(0)-G(t)
$$

is proportional to the mean-square shear stress increment. Thus, the functions $\mu_{F}(\Delta t)$ and $h(t)$ or $G(t)$ are closely related defining each other with eq. 19 or with the inverse equation 6

$$
h(t)=\frac{1}{2} \frac{\mathrm{d}^{2}}{\mathrm{~d} t^{2}}\left[t^{2} \mu_{F}(t)\right]
$$

The long-time modulus $\mu_{s f}$ was obtained as a function of temperature using the FDT relation, eq. 12 , for both systems, $768 \times 4$ and $3072 \times 4$ (cf. refs. 5 and 6). The temperature behavior of the moduli $\mu_{F}$ and $\mu_{s f}$ for the sampling time $\Delta t=10^{5}$ (in LJ units) ${ }^{44}$ is shown in Fig. 3 (the data for the larger system have been obtained in refs. 5 and 6). The glassy modulus $\mu_{s f}$ nearly vanishes at high $T$ 's (in the liquid state), while it sharply increases (and $\mu_{F}=\mu_{A}-\mu_{s f}$ decreases) as the temperature is lowered near $T_{g}$. Below $T_{g}$ (in the glassy state) $\mu_{s f}$ continues to grow, albeit rather moderately. The moduli $\mu_{F}$ and $\mu_{s f}$ are nearly the same for both systems at all temperatures apart from the low- $T$ region $(T \lesssim 0.3)$ where the smaller system shows a bit lower $\mu_{F}$ and therefore a bit higher $\mu_{s f}$. The latter low- $T$ effect is consistent with two features of stress relaxations (recall that $\mu_{F}$ and $\mu_{s f}$ are directly related to $G(t)$, cf. eqs. 19,11): (i) $G(t)$ in the 'plateau' regime is a bit higher for the smaller system; (ii) the stress relaxation time $\tau_{\alpha}$ is longer for this system below $T_{g}$ (cf. section III and note that a lower $\Delta t / \tau_{\alpha}$ always leads to a higher effective modulus $\mu_{s f}$ ).

Let us turn to the sampling time effect for the moduli. The $T$-dependencies of $\mu_{s f}$ and $\mu_{F}$ for both systems at different $\Delta t$ are depicted in Fig. 5. It is obvious that $\mu_{s f}$ decreases $\left(\mu_{F}\right.$ increases) with increasing $\Delta t$ at a given $T$ as it should be (longer relaxation leads to a lower terminal modulus $\left.\mu_{s f}\right)$. It is also clear that, as expected, the glass transition shifts to lower temperatures as $\Delta t$ 
is increased. However, the steepness of the transition (of the growth of $\mu_{s f}$ near $T_{g}$ ) does not increase with $\Delta t$ being apparently nearly constant (more precisely, the transition becomes sharper as $\Delta t$ increases from 500 to $10^{4} \mathrm{LJ}$ units, but a further increase of $\Delta t$ makes it a bit less sharp). ${ }^{45}$ This feature seems to be a curiosity of the system: at long $\Delta t$ we expect the glass transition to become progressively sharper (see the Discussion). Apparently, much longer sampling times are required to unambiguously distinguish between the theoretically expected discontinuous ${ }^{25-29}$ and continuous $^{1-5}$ scenarios of the shear modulus behavior at the glass transition.

We attempted to perform the time-temperature superposition (TTS) to collapse the $\mu_{s f}(T)$ curves for different $\Delta t$ times. The natural idea is that the glass transition occurs when the sampling time $\Delta t$ gets comparable to the characteristic time of stress relaxation $\tau_{\alpha}=\tau_{\alpha}(T)$. This concept implies that the relevant variable is $\tau_{\alpha}(T) / \Delta t$. Assuming Arrhenius (activation) behavior for the relaxation time, $\tau_{\alpha}(T)=$ const $\exp (E / T)$, where $E$ is the activation energy, we arrive at the relevant time-temperature variable $x=\frac{1}{T}+\frac{1}{E} \ln \left(\frac{\Delta t_{\text {ref }}}{\Delta t}\right)$, where $\Delta t_{\text {ref }}$ is an arbitrary reference time. A reasonable collapse of $\mu_{s f}$ vs. $x$ is achieved for $E \approx 18$ (see Fig. 5c). The same activation energy can be deduced from the temperature behavior of the shear viscosity $\eta$ near $T_{g}$ (in the range $0.37 \leq T \leq 0.41$ ) for the larger $3072 \times 4$ system (cf. Fig. 13 of ref. 6). The validity of the TTS verified in Fig. 5c is important as it supports the continuous nature of solidlike elasticity emergence at the glass transition. In fact, a continuous dependence of $T_{g}$ on $\Delta t$ (which is implied in the TTS) is incompatible with a discontinuous dependence of shear modulus, $\mu(T)$, at a finite $\Delta t$.

\section{FLUCTUATIONS AND CORRELATIONS OF $\mu_{A}$}

The modulus $\mu_{A}$ provides an instant stress response to a small instant shear. It is therefore a static, structural property of the system. The deviations of $\mu_{A}$ discussed in refs. 5 and 6 refer to the dispersion of the mean $\mu_{A}$ values time-averaged along a trajectory. More precisely, the instant affine moduli were calculated using eq. 1 at regular times separated by $\Delta t_{A}=500 \mathrm{LJ}$ units, so the mean $\mu_{A}$ is based on $n_{A}=\Delta t / \Delta t_{A}=200$ configurations for the time-window $\Delta t=10^{5} \mathrm{LJ}$. (Note that the so-called impulsive correction to $\mu_{A}$, which is proportional to $g_{n b}\left(r_{c u t}\right)$, cf. eq. 2 , was taken into account as described in ref. 11.) For better comparison, $\mu_{A}$ and its standard deviation, $\delta \mu_{A}$, were calculated in a similar way for the $768 \times 4$ system:

$$
\left(\delta \mu_{A}\right)^{2}=\left\langle\left(\tilde{\mu}_{A}-\mu_{A}\right)^{2}\right\rangle
$$


where $\tilde{\mu}_{A}$ is the mean affine modulus of a trajectory and $\mu_{A}=\left\langle\tilde{\mu}_{A}\right\rangle$ is the ensemble-averaged modulus.

The standard deviations of $\mu_{A}$ are shown for both system sizes in Fig. 6a. It is clear that $\delta \mu_{A}$ strongly increases as the system is cooled through the glass transition region. It is also obvious that $\delta \mu_{A}$ decreases with the system size. The ratio of standard deviations $\delta \mu_{A}(768) / \delta \mu_{A}(3072)$ is close to 2 at low $T \lesssim 0.35$ (cf. Fig. 6b). This fact points to the standard power-law dependence of $\delta \mu_{A}$ on the system volume: $\delta \mu_{A} \propto 1 / \sqrt{V}$ (note that the system volume is proportional to the number of particles). ${ }^{46}$ Therefore $\mu_{A}$ appears to be a self-averaging quantity whose variance decreases with the total volume as $1 / V$. This conclusion is in line with simulation studies of selfassembling networks ${ }^{30}$. In view of this property it may be tempting to relate the variance of $\mu_{A}$ with some microscopic correlation volume $V_{c}:\left(\delta \mu_{A}\right)^{2} \simeq\left(V_{c} / V\right) \mu_{A}^{2}$. What is the nature of $V_{c}$ ? Does an increase of $\delta \mu_{A}$ at low $T$ signify an increase of a structural correlation volume? These and other questions related to the statistics of $\mu_{A}$ are discussed below in this section.

It is worth stressing that the statistics of the time-averaged $\mu_{A}$ involve time-correlations between instant $\mu_{A}$ along the trajectory. These correlations are dynamical (rather than solely structural) in nature and therefore strongly depend on temperature. To avoid the dynamical aspect, one has to consider directly the statistics of the instant moduli $\tilde{\mu}_{A}\left(n_{A} m\right.$ values in total for $m=100$ independent trajectories). The corresponding standard deviation is denoted here as $\delta_{1} \mu_{A}$; it was calculated based on 100 independent trajectories for each $T$. We analysed separately the two non-ideal (excess) contributions to $\mu_{A}$, one due to interactions between bonded monomers $\left(\mu_{A b}\right)$ and another due to LJ-interactions $\left(\mu_{A l j}\right)$. The overall $\mu_{A}$ is a sum of $\mu_{A b}, \mu_{A l j}$ and the ideal-gas term $c T$ ( $c=N_{m} / V$; the ideal term contributes only $\sim 1 \%$ to $\mu_{A}$; its fluctuations are totally negligible). The results for the $768 \times 4$ system are shown in Fig. 7a. One can observe that the mean $\mu_{A}, \mu_{A b}$ and $\mu_{A l j}$ all increase rather weakly as $T$ is lowered (cf. Fig. 7a). Being static properties the instantaneous $\mu_{A}$-moduli are expected to show a cusplike feature at the glass transition in analogy with the $T$-dependence of density (cf. Fig. 1). Such features (weak virtual cusp near $T_{g}$ ) are indeed visible in the $T$-dependencies of $\mu_{A}, \mu_{A b}$ and $\mu_{A l j}$ (cf. Fig. 7a).

Remarkably, however, the standard deviations (across the $m n_{A}$-ensemble) of the instant values of the $\mu_{A}$-moduli are nearly independent of $T$ (cf. Fig. 7b) in drastic contrast with a strong increase of $\delta \mu_{A}$ (deviations of the time-averaged $\mu_{A}$ ) near and below $T_{g}$ (cf. Fig. 7d). Note that $\delta_{1} \mu_{A} \approx \delta \mu_{A}$ for $T \lesssim 0.36$, but $\delta_{1} \mu_{A} \gg \delta \mu_{A}$ for $T \gtrsim 0.4$. It means that the amorphous structure stays largely frozen at low $T$ (so, in particular, bond-orientational fluctuations are suppressed), and therefore 
the fluctuations of $\mu_{A}$ along the time-trajectory become negligible in this regime (as the system rests in a virtually single inherent structure within a metabasin). We also observe that generally $\mu_{A}$ is dominated by the bond contribution, $\mu_{A b}$. A similar statement is even more true for the standard deviations: the total deviation $\delta_{1} \mu_{A}$ is nearly equal to that for bonds, $\delta_{1} \mu_{A b}$.

We obtained and compared contributions to the dispersion of the instant $\mu_{A}$ due to thermal fluctuations along the trajectory, $\operatorname{var}_{F}\left(\mu_{A}\right)$, and due to permanent variations between independently cooled configurations (metabasins), $\operatorname{var}_{M B}\left(\mu_{A}\right):\left(\delta_{1} \mu_{A}\right)^{2}=\operatorname{var}_{F}\left(\mu_{A}\right)+\operatorname{var}_{M B}\left(\mu_{A}\right)$. Quite expectedly we found that the MB-contribution dominates well below $T_{g}$ (at $T \lesssim 0.3$ ), while both contributions are comparable near $T_{g}$ (and, of course, the fluctuation contribution dominates above $\left.T_{g}\right)$.

One may wonder if a structural correlation length $\xi_{s}$ can be estimated based on these results. Below we show that this is not really possible. The point is that both $\mu_{A b}$ and $\delta_{1} \mu_{A b}$ (providing dominant contributions to the affine modulus and its standard deviation) can be predicted assuming no correlations between the polymer bonds. This assumption is reasonable since bonding interactions are much stronger than $\mathrm{LJ}$ interactions in our simulation model with $k_{\text {bond }}=1110$ in $\mathrm{LJ}$ units. (A renormalization of $k_{\text {bond }}$ due to $\mathrm{LJ}$ interactions can be neglected for the same reason.) It leads to the following results (small corrections of relative order $T /\left(k_{\text {bond }} l_{\text {bond }}^{2}\right)$ are neglected here):

$$
\mu_{A b} \approx \frac{1}{20} c k_{\text {bond }} l_{\text {bond }}^{2}, \delta_{1} \mu_{A b} \approx \frac{1}{5} \sqrt{\frac{2}{21}} c k_{\text {bond }} l_{\text {bond }}^{2} / \sqrt{N_{m}}, \frac{\delta_{1} \mu_{A b}}{\mu_{A b}} \approx 4 \sqrt{\frac{2}{21 N_{m}}}
$$

These theoretical results are also shown in Fig. 7a-d. The relative deviations of $\mu_{A b}$ are thus predicted to be $T$-independent: $\frac{\delta_{1} \mu_{A b}}{\mu_{A b}} \approx 0.0223$ for $N_{m}=768 \times 4$, while simulations point to $\approx 0.025$ for this ratio (cf. Figs. 7c and 8). It is clear that the predictions are generally in good agreement with our simulation data: the theory just slightly overestimates $\mu_{A b}$ and underestimates $\delta_{1} \mu_{A b}$. Given that $\delta_{1} \mu_{A} \approx \delta_{1} \mu_{A b}$ (cf. Fig. 7b), we conclude that structural correlations cannot be resolved based on fluctuations of instant $\mu_{A}$ for the model we consider. In other words, $\mu_{A}$ does not seem to be an appropriate variable to probe the correlation length $\xi_{s}$. As for the effective correlation volume $V_{c}$, it always corresponds to about 1 particle (monomer) independent of temperature and the system size (cf. eq. 22).

The revealed $T$-independence of $\delta_{1} \mu_{A}$ invites the question: why the deviations $\delta \mu_{A}$ of the timeaveraged $\mu_{A}$ depend on $T$ so strongly (they increase by a factor of $\sim 20$ between $T=0.55$ and 0.05)? The reason is that while $\mu_{A}$ is always averaged over $n_{A}=200$ transient configurations along 
each trajectory, these instant states are independent at $T=0.55$ (where relaxation time $\tau_{\alpha}$ is much shorter than the time interval $\Delta t_{A}$ between the configurations), but they are strongly correlated for $T=0.05\left(\tau_{\alpha} \gg \Delta t_{A}\right)$. As a result $\delta \mu_{A}$ is smaller than $\delta_{1} \mu_{A}$ by a factor $1 / \sqrt{n_{A}}$ at high $T$ 's, but this reduction is not applicable at low $T$ 's where $\delta \mu_{A} \sim \delta_{1} \mu_{A}$ (cf. Figs. 7d and 8).

So far the instant (or time-averaged) $\mu_{A}$ was calculated for a given fixed shear plane (say, $x y$ ). The instant $\mu_{A}$ was thus calculated using the general equations 1,2 (cf. refs. 6, 11, and 31). Recalling the macroscopic isotropy of the system, we tried a different approach to obtain instant $\mu_{A}$ using pre-averaging over all possible shear planes. The resultant expression for the pre-averaged instant $\mu_{A}$ does not involve bond orientations and can be conveniently written in terms of pair correlation functions like $g(r)$, the Kirkwood radial distribution function:

$$
\mu_{A}=\mu_{A l j}+\mu_{A b}+c T
$$

where

$$
\begin{gathered}
\mu_{A l j}=A_{d} c^{2}\left\{\int_{0}^{r_{c u t}}\left[r u_{L J}^{\prime \prime}(r)+(d+1) u_{L J}^{\prime}(r)\right] g_{n b}(r) r^{d} \mathrm{~d} r-\left[r^{d+1} u_{L J}^{\prime}(r) g_{n b}(r)\right]_{r=r_{c u t}}\right\} \\
\mu_{A b}=A_{d} c^{2} \int_{0}^{r_{c u t}}\left[r u_{b}^{\prime \prime}(r)+(d+1) u_{b}^{\prime}(r)\right] g_{b}(r) r^{d} \mathrm{~d} r
\end{gathered}
$$

Here $d=3$ is the space dimension, $A_{d}=\frac{\pi^{d / 2}}{4 \Gamma(d / 2+2)}=\frac{2 \pi}{15}, u_{b}(r)=0.5 k_{b o n d}\left(r-l_{\text {bond }}\right)^{2}$ is the interaction potential for bonded monomers, $u_{L J}(r)=4\left(r^{-12}-r^{-6}\right)$ is the LJ potential in LJ units, $g_{b}(r)$ is the pair correlation function for bonded monomers (analogous to the Kirkwood function), and $g_{n b}(r)=g(r)-g_{b}(r)$. Note that

$$
\int g_{b}(r) \mathrm{d}^{d} r=\frac{2(N-1)}{N c}=\frac{3}{2 c} \text { with } N=4
$$

In practice the two integrals in eqs. 24 are replaced by sums according to the rule:

$$
A_{d} c^{2} \int_{0}^{r_{c u t}} X(r) g_{\alpha}(r) r^{d} \mathrm{~d} r \rightarrow \frac{1}{d(d+2)} \frac{1}{V} \sum_{l(\alpha)} X\left(r_{l}\right)
$$

where $\alpha$ is either ' $\mathrm{b}$ ' (polymer bond) or ' $\mathrm{nb}$ ' (LJ interaction), $l(\alpha)$ runs over all (disordered) monomer pairs of type $\alpha$, and $X(r)$ is any function.

We found that the orientation-averaging dramatically reduces the variance of $\mu_{A}$ : both standard deviations $\delta \mu_{A}$ and $\delta_{1} \mu_{A}$ (of time-averaged and instant $\mu_{A}$ values, respectively) decrease as a result by a factor changing from $\sim 6$ to $\sim 20$ as $T$ is lowered from 0.55 to 0.05 (see Figs. 7e,f). 
This unexpectedly strong effect has a simple physical meaning: the variance of $\mu_{A}$ is dominated by bond-orientational fluctuations which are effectively eliminated in the new definition, eqs. 23, 24. On more quantitative grounds, this effect can be analyzed for the dominant bondrelated part of $\mu_{A}$. Fluctuations of the pre-averaged $\mu_{A b}$ defined in eqs. 23, 24 are due to bondlength fluctuations which are much weaker than orientational fluctuations. Assuming (as before) independent bonds we found:

$$
\delta_{1} \mu_{A b(\text { or })} \approx 0.2 c l_{\text {bond }} \sqrt{\frac{3 T k_{b o n d}}{N_{m}}}
$$

The standard deviation of instant but orientation-averaged $\mu_{A b}$ is thus expected to decrease as $\sqrt{T}$ at low temperatures (following the amplitude of bond-length fluctuations). This prediction is in very good agreement with simulation results for $\delta_{1} \mu_{A b(o r)}$ in the whole studied $T$-range (cf. dashed black and magenta curves in Fig. 7e).

Comparing eq. 25 with eq. 22 we observe that the standard deviation of $\mu_{A b}$ is now significantly reduced by a large factor $\frac{\delta_{1} \mu_{A b}}{\delta_{1} \mu_{A b(o r)}}=\frac{l_{\text {bond }}}{3} \sqrt{\frac{2 k_{b o n d}}{7 T}} \approx \frac{5.74}{\sqrt{T}}$ in quantitative agreement with simulation results and in qualitative agreement with a similar reduction of $\delta_{1} \mu_{A}$ and $\delta \mu_{A}$ (deviations of instant and time-averaged $\mu_{A}$, cf. Fig. 7f).

Note that fast increase of the ratio of the time-averaged deviations, $\delta \mu_{A} / \delta \mu_{A(\text { or })}$ near $T_{g}$ (cf. Fig. 7f) is due to an increase of the orientational relaxation time (leading to a poorer self-averaging of $\mu_{A}$ over the sampling time at $T<T_{g}$, hence an increase of $\delta \mu_{A}$ ), while a decrease of the same ratio at lower $T<0.3$ is due to a slowdown (partial freezing) of bond-length fluctuations leading to a poorer self-averaging of $\mu_{A(o r)}$, hence an increase of the denominator, $\delta \mu_{A(o r)}$, on further cooling.

To conclude, eqs. 23, 24 are useful to obtain more precise instantaneous affine shear modulus $\mu_{A}$. With the standard definition of $\mu_{A}$ (without orientational pre-averaging), the standard deviation $\delta \mu_{A}$ becomes comparable to $\delta \mu_{F}$ at low $T$ (in particular, for the smaller 769x4 system, cp. figures 7e and 9a), so the standard deviation of $\mu_{s f}=\mu_{A}-\mu_{F}$ is somewhat larger than $\delta \mu_{F}$. Using $\mu_{A}$ obtained with eqs. 23,24 we arrive at less fluctuating $\mu_{s f}$ with $\delta \mu_{s f} \approx \delta \mu_{F}$ at all $T$ 's since fluctuations of $\mu_{A}$ in this case are always totally negligible. 


\section{DISPERSION OF $\mu_{F}$}

The fluctuation modulus $\tilde{\mu}_{F}$ (cf. eq. 18) is a random variable changing across the $m n_{A^{-}}$ ensemble; its standard deviation $\delta \mu_{F}$ (with a large ensemble, $m \gg 1$ ) is defined in analogy with $\delta \mu_{A}$ :

$$
\left(\delta \mu_{F}\right)^{2}=\left\langle\left(\tilde{\mu}_{F}-\mu_{F}\right)^{2}\right\rangle
$$

where $\mu_{F}$ is the mean value defined in eq. 16. The simulation results for the deviations $\delta \mu_{F}$ at different temperatures are shown for both systems (at $\Delta t=10^{5}$ ) in Fig. 9a. The data for the $3072 \times 4$ system have been discussed in refs. 5 and 6 . It was reported there that both $\delta \mu_{F}$ and $\delta \mu_{s f} \approx \delta \mu_{F}$ (this equation comes from eq. 12 and the fact that the variance of $\mu_{A}$ can be neglected with respect to the variance of $\mu_{F}$, cf. the previous section $V$ and ref. 6) show a peak near the apparent glass transition temperature $T_{g}$. This feature is confirmed here for the new $768 \times 4$ system. The peaks are located at the same temperature $T \approx 0.36$. It is interesting that the peak height is nearly independent of the system volume (the peak is just a bit higher for the smaller system) and that the deviations, $\delta \mu_{F}$, are identical for both systems in the liquid regime $\left(T>T_{g}\right)$. On the other hand, at low temperatures $\delta \mu_{F}$ is significantly larger for the smaller system (as compared to the larger one).

The sampling time effect on $\delta \mu_{F}$ is illustrated in Fig. 10a. In the liquid regime $\delta \mu_{F}$ significantly increases as $\Delta t$ is shortened (this behavior is in accord with an increase of effective $T_{g}$ for shorter $\Delta t$, cf. end of section IV). ${ }^{47}$ By contrast, $\delta \mu_{F}$ decreases (albeit rather moderately) for shorter $\Delta t$ in the peak region and below the transition. This tendency (an increase of $\delta \mu_{F}$ with $\Delta t$ ) seem to weaken at low $T$ and disappear at the lowest $T=0.05$.

The discovered peak of $\delta \mu_{F}$ near $T_{g}$ is a remarkable feature ${ }^{5,6}$ demanding an explanation. A qualitative argument elucidating this behavior is presented in the Discussion. Below, instead, we describe a quantitative approach predicting $\delta \mu_{F}$ based on the known relaxation modulus $G(t)$.

The fluctuation modulus for a given trajectory, $\tilde{\mu}_{F}$, is directly related to the stress function $\sigma(t)$, cf. eq. 18 , which is a stochastic process characterized by some stationary probability distribution enveloping all systems of the statistical ensemble we consider. The basic assumption adopted here is that this distribution is nearly Gaussian (i.e., $\sigma(t)$ is a Gaussian process). Its validity is discussed in the next section. It is instructive to consider a discrete version of the theory involving arrays $\sigma_{i} \equiv \sigma\left(t_{i}\right)$ of stress recorded at times $t_{i}=i \delta t$, where $\delta t$ is the time interval between successive stress calculations ( $\delta t=0.05$ in our simulations), and $i$ is an integer changing from 1 to $I=\Delta t / \delta t$. 
Then

$$
\tilde{\mu}_{F}=\frac{V}{T}\left(I^{-1} \sum_{i} \sigma_{i}^{2}-I^{-2} \sum_{i, j} \sigma_{i} \sigma_{j}\right)
$$

where $j$ is also changing from 1 to $I$. The mean value of $\tilde{\mu}_{F}$ can be obtained noting that

$$
\frac{V}{T}\left\langle\sigma_{i} \sigma_{j}\right\rangle=G_{i-j}+\text { const }
$$

with $G_{i-j} \equiv G\left(\left|t_{i}-t_{j}\right|\right)$ :

$$
\mu_{F}=G_{0}\left(1-I^{-1}\right)-2 I^{-2} \sum_{s=1}^{I-1}(I-s) G_{s}
$$

The latter equation can be considered as the discrete version of eq. 19. The variance of $\mu_{F}$ is

$$
\left(\delta \mu_{F}\right)^{2}=\left\langle\left(\tilde{\mu}_{F}\right)^{2}\right\rangle-\mu_{F}^{2}
$$

The r.h.s. of the above equation involves terms like $\left\langle\sigma_{i} \sigma_{j} \sigma_{i^{\prime}} \sigma_{j^{\prime}}\right\rangle$ (emerging on recalling eq. 27). For a Gaussian $\sigma(t)$ such quartic correlators are reduced to pair correlators

$$
\left\langle\sigma_{i} \sigma_{j} \sigma_{i^{\prime}} \sigma_{j^{\prime}}\right\rangle=\left\langle\sigma_{i} \sigma_{j}\right\rangle\left\langle\sigma_{i^{\prime}} \sigma_{j^{\prime}}\right\rangle+\ldots
$$

which are related to $G(t)$ via eq. 28 . As a result we get

$$
\left(\delta \mu_{F}^{(G)}\right)^{2}=\frac{2}{I^{4}}\left\{\left[\sum_{i, j} G_{i-j}\right]^{2}+I^{2} \sum_{i, j} G_{i-j}^{2}-2 I \sum_{i, j, s} G_{s-i} G_{s-j}\right\}
$$

The superscript ' $\mathrm{G}$ ' here means that eq. 30 gives the variance of $\mu_{F}$ using the Gaussian approximation.

The 'Gaussian' standard deviations, $\delta \mu_{F}^{(G)}$, were calculated for both systems and different $T$ 's and sampling times $\Delta t$. (In practice, all the multiple sums in eq. 30 were reduced to single sums to accelerate the calculation.) The obtained results are compared with simulation data for $\delta \mu_{F}$ in Fig. 9b. Noteworthily, the $T$-dependencies of the 'Gaussian' deviations $\delta \mu_{F}^{(G)}$ are nearly the same for both systems. One can observe an excellent agreement between $\delta \mu_{F}^{(G)}$ and $\delta \mu_{F}$ in the liquid regime: $\delta \mu_{F} \approx \delta \mu_{F}^{(G)}$ at $T>T_{g}$ for both systems. Moreover, the Gaussian approximation correctly reproduces the simulation data also in the peak region (for $T \gtrsim 0.3$ ) for the larger system, while for the $768 \times 4$ system the peak height is somewhat underestimated by the theory. At low temperatures, $T<0.3$, the predicted $\delta \mu_{F}^{(G)}$ strongly decreases while $\delta \mu_{F}$ seems to saturate at a finite level. 
The above conclusions are supported with Fig. 10b illustrating the temperature dependencies of $\delta \mu_{F}$ and $\delta \mu_{F}^{(G)}$ at different sampling times $\Delta t$. At low $T<T_{g}$ the effect of $\Delta t$ is complicated: at $T \gtrsim 0.2$ the theoretical deviation $\delta \mu_{F}^{(G)}$ increases with $\Delta t$, while the opposite tendency works at lower temperatures $(T \lesssim 0.15)$. (Note that $\delta \mu_{F}$ seems to exhibit a qualitatively similar behavior, albeit with a crossover at a lower $T \sim 0.05$, cf. Fig. 10a.) Where such behavior could originate from? It is relatively easy to clarify this point for $\delta \mu_{F}^{(G)}$. The analysis can be based on the following equation stemming directly from eq. 30

$$
\left(\delta \mu_{F}^{(G)}\right)^{2}=\frac{1}{2 I^{4}} \sum_{i j i^{\prime} j^{\prime}}\left(G_{i-j}+G_{i^{\prime}-j^{\prime}}-G_{i-j^{\prime}}-G_{j-i^{\prime}}\right)^{2}
$$

At low $T$ 's the lion's share of time points falls into the plateau regime where $G$ is nearly constant (cf. Fig. 4), hence the summand is small: its typical value is $\left(G(\Delta t)-G(\Delta t / 2)^{2} \sim g^{2}\right.$, where $g=(\partial G / \partial \ln t)_{t \sim \Delta t}$. The long-time contribution to the variance, $\left(\delta \mu_{F}^{(G)}\right)^{2}$, is therefore $\sim g^{2}$ with small $g=g(T, \Delta t)(g \lesssim 1$ for $T<0.3)$. When 2 time points (say, $i$ and $j)$ get close to each other, the summand can take a much larger value $\left(\sim\left(\mu_{A}-\mu_{s f}\right)^{2}=\mu_{F}^{2}\right)$, but the 'probability' of such an event is low, $\sim \tau_{0} / \Delta t$. Taking into account both contributions we write

$$
\left(\delta \mu_{F}^{(G)}\right)^{2} \sim g(T, \Delta t)^{2}+\mu_{F}^{2} \tau_{0} / \Delta t, \Delta t>\tau_{0}
$$

where $\tau_{0}$ is the time-scale of initial fast stress relaxation (before the plateau regime). The $g$-factor here increases with $\Delta t$, but this increase becomes extremely weak at low $T$ (cf. Fig. 4). By contrast, the second term decreases with $\Delta t$ (for $\Delta t>\tau_{0}$ ) and is nearly independent of temperature. It wins at very low $T$ 's where the $g$-variation can be neglected thus leading to a decrease of the whole variance $\left(\delta \mu_{F}^{(G)}\right)^{2}$ with $\Delta t$.

It is interesting that the $\Delta t$-dependence of $\delta \mu_{F}^{(G)}$ for $\Delta t \gg \tau_{0}$ is qualitatively similar to its $T$ dependence (the main relevant parameter is $\Delta t / \tau_{\alpha}$ which increases either with $\Delta t$ or with $T$ ). Both $\mu_{F}$ and $\delta \mu_{F}^{(G)}$ obviously vanish at $\Delta t=0$. At high temperatures (above $T_{g}$ ) the Gaussian deviation $\delta \mu_{F}^{(G)}$ first increases with $\Delta t$ at the time-scale $\Delta t \lesssim \tau_{\alpha}$ and then decreases at longer $\Delta t$. At low $T$ $\left(T \lesssim T_{g}\right)$ the stress relaxation proceeds in two steps with times $\sim \tau_{0}$ and $\sim \tau_{\alpha}$, and we predict 2 peaks of $\delta \mu_{F}^{(G)}$ vs. $\Delta t$ : one is related to $\Delta t \sim \tau_{0}$ and the other to much longer time, $\Delta t \sim \tau_{\alpha}$.

To sum up, it appears that the Gaussian theory works well above $T_{g}$ and in the transition (peak) region, but fails at low $T$ 's. In the next Discussion section we explain this behavior and deduce some important information stemming from it. 


\section{DISCUSSION}

\section{A. Solidification transition}

Our analysis shows (cf. section IV) that the transition from liquid to amorphous solid behavior (a nearly steplike increase of the static shear modulus $\mu_{s f}$ ) occurs in the $T$-region where the terminal relaxation time is comparable with the sampling time, $\tau_{\alpha}(T) \sim \Delta t$ (for long enough $\Delta t$ this regime involves a long-time plateau in the shear relaxation modulus $G(t)$ ). The latter condition defines the apparent glass transition temperature $T_{g}=T_{g}(\Delta t)$ which depends on the explored time-window $\Delta t$ and corresponds to both the steepest increase of $\mu_{s f}=\mu_{s f}(T)$ (cf. Fig. 5b) and the maximum of its standard deviation $\delta \mu_{s f} \approx \delta \mu_{F}$ (cf. Fig. 10a).

In terms of relaxation functions like $G(t)$ the vitrification can be considered as a transition from the glassy plateau regime to the liquid regime with vanishing $G(t)$. This transition occurs at $t \sim \tau_{\alpha}(T)$, more precisely, in the region where $G(t) / G\left(\tau_{\alpha}\right) \sim 1$. Assuming the KWW stretched exponential relaxation law $^{24}$ for $G(t)$ it leads to the time-region whose width in log-scale is defined by $\delta\left(\ln \left(t / \tau_{\alpha}(T)\right)\right) \sim 1 / \beta$, where $\beta$ is the stretching exponent. Therefore, the temperature width, $\delta T_{g}$, of the glass-transition region (for a given time-window $\Delta t$ ) can be roughly defined by the condition $\delta\left(\ln \left(\tau_{\alpha}(T) / \Delta t\right)\right) \sim 1 / \beta$ leading to $\delta T_{g}\left|\partial \ln \tau_{\alpha} / \partial T\right|_{T=T_{g}} \sim 1 / \beta$. The latter estimate can be rewritten as

$$
\delta T_{g} / T_{g} \sim 1 /\left(\beta m_{f}\right)
$$

where $m_{f}=-\partial \ln \tau_{\alpha} /\left.\partial \ln T\right|_{T=T_{g}}$ is the fragility index (a similar dependence of $\delta T_{g}$ on $m_{f}$ was predicted in the review ${ }^{41}$, cf. eq. (4.5a) there). An Arrhenius increase of the relaxation time below $T_{g}, \tau_{\alpha} \sim \tau_{0} \exp (E / T)\left(\tau_{0}\right.$ is the time-scale of particle collisions), leads to $m_{f} \sim \ln \left(\tau_{\alpha} / \tau_{0}\right) \sim$ $\ln \left(\Delta t / \tau_{0}\right)$. Hence, the transition width $\delta T_{g} / T_{g}$ is expected to logarithmically decrease with the sampling time $\Delta t$ :

$$
\delta T_{g} / T_{g} \propto 1 / \ln \left(\Delta t / \tau_{0}\right)
$$

This decrease is very slow, and perhaps this is the reason why it is not apparent in the simulation data (cf. Fig. 5b). (Note that in the case of a super-Arrhenius increase of $\tau_{\alpha}, \tau_{\alpha} \sim$ $\tau_{0} \exp \left(E /\left(T-T_{0}\right)\right)$ (the VFT law), the fragility index shows a faster, but still logarithmic dependence on $\tau_{\alpha}$.) Theoretically, there is no doubt that the transition width must vanish in the limit $\Delta t \rightarrow \infty$ as long as the glassy plateau ${ }^{24}$ persists at however low temperatures (and we are not aware of any data pointing to the contrary). In this case the glass transition becomes asymptotically dis- 
continuous as $\Delta t \rightarrow \infty$, but the main question is whether the amorphous state is possibly stable at the transition temperature $T_{g}(\Delta t)$ in this limit. Its discussion is outside the scope of the present paper.

\section{B. Dispersion of $\mu_{A}$}

In section $\mathrm{V}$ we analyzed separately the $\mathrm{LJ}$ and chemical bond contributions to the affine shear modulus $\mu_{A}$. It was shown that orientational preaveraging leads to a strong decrease of $\delta \mu_{A}$, the standard deviation of $\mu_{A}$. This effect elucidates an important role of bond orientational fluctuations for $\delta \mu_{A}$.

It is also remarkable that while the fluctuations of the total instant orientation-averaged $\mu_{A}$ get weaker at low $T$ roughly in parallel with those for the chemical bond contribution $\left(\mu_{A b}\right)$, the analogous fluctuations of the LJ-contribution, $\mu_{A l j(\text { or })}$, show a stronger decay right below $T_{g}$ (see Fig. 7e: the green curve for $\delta_{1} \mu_{A l j(o r)}$ shows a downward cusp at $T_{g}$ ). What's the physical meaning of this feature? The modulus $\mu_{A l j(o r)}$ is sensitive to distances between the neighboring particles, so the behavior of $\delta_{1} \mu_{A l j(o r)}$ (note that this quantity is a static property) indicates that the amorphous glassy structures below $T_{g}$ involve progressively weaker dispersion of inter-particle distances as $T$ is decreased (a similar structural effect for chemical bonds is much less pronounced due to their high rigidity $\left.k_{\text {bond }}\right)$.

Another interesting feature concerns the behavior of $\delta \mu_{A}$ and $\delta \mu_{A(o r)}$ shown in Fig. 7e (cf. black and red curves): both deviations show a sharp increase as $T$ is lowered in the glass-transition region, however the increase of $\delta \mu_{A}$ (by a factor of 10) is much stronger than that for $\delta \mu_{A(o r)}$ (roughly by a factor of $\sqrt{10}$ ). This difference can be explained as follows: $\mu_{A}$ fluctuations are mainly due to rotations of chemical bonds, which get virtually frozen below $T_{g}$ leading to a poorer self-averaging of $\mu_{A}$ (hence, to a significant increase of $\delta \mu_{A}$ ). By contrast, $\mu_{A(\text { or })}$ is insensitive to bond orientations: rather its fluctuations are defined by the bond-length dynamics which become only partially constrained right below $T_{g}$ (leading to a weaker increase of $\delta \mu_{A(o r)}$ ). It is also likely that bond-length fluctuations get progressively more restricted at lower $T$ leading to a further increase of $\delta \mu_{A(\text { or })}$ (and, hence, to a decrease of the ratio $\delta \mu_{A} / \delta \mu_{A(\text { or })}$, cf. Fig. 7f). The same effect also results in finite levels of both $\delta \mu_{A b(\text { or })}$ and $\delta_{1} \mu_{A b(\text { or })}$ (and, of course, of $\delta \mu_{A(\text { or })}$ and $\delta_{1} \mu_{A(\text { or })}$ ) at $T \rightarrow 0$ since not only bond orientational heterogeneities but also bond-length fluctuations must be arrested in this limit. 


\section{Peaks of $\mu_{F}$ and $\delta \mu_{F}$}

The fluctuation modulus $\mu_{F}$ shows a peak near $T_{g}$ (cf. Figs. 3a, 5a). This behavior can be clarified in a simple way. At high temperatures (above $T_{g}$ ) the terminal shear modulus $\mu \approx \mu_{s f}$ is close to 0 , so $\mu_{F} \approx \mu_{A}$ by virtue of eq. 12 . As $T$ decreases at constant pressure, the instantaneous modulus $\mu_{A}$ increases due to stronger interactions between particles: the density (monomer concentration $c$ ) of the system increases, hence the mean interparticle distance decreases leading to a higher interaction energy at lower $T$; this interaction contribution to $\mu_{A}$ typically overwhelms the momentum contribution which is equal to $c T$. By contrast, at low $T \lesssim T_{g}$ the effective modulus $\mu_{s f}$ starts to grow rapidly (the solidification transition). This increase is stronger than the moderate increase of $\mu_{A}$, hence $\mu_{F}=\mu_{A}-\mu_{s f}$ decreases as the system is cooled below $T_{g}$ (this tendency also comes from a simple observation that the system dynamics slow down at low $T$, hence the drop of $G(t)$ during the same time $t \sim \Delta t$ becomes weaker). As a result $\mu_{F}$ as a function of $T$ (at a constant sampling time $\Delta t$ ) develops a peak near $T_{g}$.

Let us turn to the variance of $\mu_{F}$, defined in eq. 26 , which is nearly equal to the variance of $\mu_{s f}$ (cf. section VI) and shows a sharp peak near $T_{g}$ (cf. Fig. 9a). A qualitative explanation of this behavior is given below: At high temperatures $\left(T>T_{g}\right)$, in the liquid regime the fluctuation modulus $\tilde{\mu}_{F}$ is dominated by the term $\tilde{\mu}_{0}$ in eq. 17 (since $\bar{\sigma}$ is strongly suppressed by self-averaging to 0$)$ :

$$
\tilde{\mu}_{F} \approx \tilde{\mu}_{0}=\mathrm{const} \int_{0}^{\Delta t} \sigma(t)^{2} \mathrm{~d} t
$$

The stresses $\sigma\left(t_{1}\right)$ and $\sigma\left(t_{2}\right)$ are virtually uncorrelated if $\left|t_{1}-t_{2}\right| \gtrsim \tau_{\alpha}$, where $\tau_{\alpha}$ is the terminal (longest) stress relaxation time. Above $T_{g}$ the time $\tau_{\alpha}$ is short, $\tau_{\alpha} \ll \Delta t$, hence the integral in eq. 33 can be considered as a sum of many $(K)$ uncorrelated similar contributions, $K=\Delta t / \tau_{\alpha} \gg 1$. As a result $\tilde{\mu}_{F}$ efficiently self-averages in this regime: its variance is small being inversely proportional to $K$ :

$$
\left(\delta \mu_{F}\right)^{2} / \mu_{F}^{2} \sim 1 / K=\tau_{\alpha} / \Delta t
$$

As a matter of fact, that sort of argument (to get eq. 34) is well-known in the simulation literature (cf. section 4.2.4 of ref. 33 showing that finite sampling time effects may lead to statistical and systematic errors of numerical results). Note that the above equation resembles eq. 32 where $\tau_{0}$ is replaced by $\tau_{\alpha}$ and the first term is omitted (being negligible) as there is no plateau in the liquid regime. Therefore, here $\delta \mu_{F} \propto \sqrt{\tau_{\alpha}}$, and so the standard deviation $\delta \mu_{F}$ increases significantly as the system is cooled towards $T_{g}$, following the behavior of the relaxation time $\tau_{\alpha}=\tau_{\alpha}(T)$. 
Quantitatively, $\delta \mu_{F}$ in the liquid regime (where $\tau_{\alpha} \ll \Delta t$ ) is accurately predicted with the Gaussian approximation (cf. eqs. 30, 31) giving

$$
\left(\delta \mu_{F}\right)^{2} \simeq 4 \eta_{2} / \Delta t
$$

where

$$
\eta_{2}=\int_{0}^{\infty} G(t)^{2} \mathrm{~d} t
$$

Turning in passing to the terminal modulus $\mu_{s f}$, recall that $\mu_{s f} \simeq 2 \eta / \Delta t$ for $\Delta t \gg \tau_{\alpha}$ (cf. eq. 11 and ref. 6), where $\eta=\int_{0}^{\infty} G(t) \mathrm{d} t$ is the shear viscosity. Therefrom we find the relative variance of $\mu_{s f}$ (also recalling that $\delta \mu_{s f} \simeq \delta \mu_{F}$ )

$$
\left(\delta \mu_{s f} / \mu_{s f}\right)^{2} \simeq\left(\eta_{2} / \eta^{2}\right) \Delta t \sim \Delta t / \tau_{\alpha}
$$

which is increasing with the time-window $\Delta t$ in the liquid regime.

At $T<T_{g}$ the system enters the glassy regime where the terminal relaxation time is very long, $\tau_{\alpha} \gg \Delta t$. As a result, the time $\tau_{\alpha}$ becomes irrelevant for $\delta \mu_{F}$. Close to $T_{g}$ (but below it) the standard deviation $\delta \mu_{F}$ is still well-described by the Gaussian approximation (cf. Figs. 9b, 10b), so we can make use of eq. 32. The second term in this equation is nearly constant below $T_{g}$ (for a given $\Delta t$ and $\left.\tau_{0} \ll \Delta t\right)$, while the first term involving $g(T, \Delta t)=(\partial G / \partial \ln t)_{t \sim \Delta t}$ strongly decreases as the system is further cooled below $T_{g}$ (cf. Fig. 4).

To sum up, the arguments presented above show that $\delta \mu_{F}$ must strongly diminish as $T$ deviates from $T_{g}$ getting either cooler or warmer, thus producing a sharp peak near $T_{g}$.

The dependencies of $\delta \mu_{s f} \approx \delta \mu_{F}$ and $\mu_{s f}$ on $T$ are depicted in Fig. 12. In is obvious that $\delta \mu_{s f} \sim \mu_{s f}$ near the peak of $\delta \mu_{s f}(T):$ the fluctuations of the long-time shear modulus across the ensemble are of the same order as its average over all the independent configurations, so the mean $\mu_{s f}$ is not necessarily a good reporter of the typical system behavior near the glass transition. This important finding was mentioned in the previous papers ${ }^{5,6}$ for the $3072 \times 4$ system. It is now clear that this feature is general. It is also supported by the Gaussian theory: eq. 31 shows that $\delta \mu_{F}$ is roughly equal to the typical change of $G(t)$ in the region $t \sim \Delta t$ (say, between $t_{1} \sim \Delta t / 2$ and $t_{2} \sim \Delta t$ ); this change is comparable to $\mu_{s f}$ for $\Delta t \sim \tau_{\alpha}$ (that is, near the glass transition, at the peak). Noteworthily, the relation $\delta \mu_{s f} \sim \mu_{s f}$ also comes from eq. 35 with $\Delta t \sim \tau_{\alpha}$. 


\section{Gaussian approximation}

We developed a theory of $\mu_{F}$ fluctuations assuming the Gaussian statistics for the instant stress $\sigma(t)$ (cf. section VI). This approach can be applied to analyze fluctuations of other properties. As an example let us consider the mean-square of the time-averaged stress, $\mu_{1}=\frac{V}{T}\left\langle\bar{\sigma}^{2}\right\rangle$ (cf. eq. 13), which is equal to $\mu_{s f}$ for well equilibrated systems (at $T \gtrsim 0.3$ ). The Gaussian theory predicts the following universal relation

$$
\delta \mu_{1}=\sqrt{2} \mu_{1}
$$

This prediction is verified by our simulation data as presented in Fig. 13. It shows that in this case the Gaussian approximation works also at low $T$ 's: its failure to correctly predict $\delta \mu_{F}$ at $T \lesssim 0.3$ is related to the fact that the Gaussian variance of $\mu_{F}$ strongly decreases at low $T$ 's while the normally subdominant correction stays finite.

\section{E. Finite size effects and dispersion of $\mu_{F}$ and $\mu_{s f}$}

The results shown in Fig. 9b indicate that (i) the dispersion of $\mu_{F}$ at $T \gtrsim T_{g}$ (in the liquid regime and near the glass transition) is well described by the Gaussian approximation, and (ii) $\delta \mu_{F}$ nearly does not depend on the system size in this regime. The first observation means that the statistics of stress fluctuations is likely to be nearly Gaussian at $T \gtrsim 0.35$, which is quite natural for the liquid regime. The second point simply follows from the first one (that $\delta \mu_{F} \approx \delta \mu_{F}^{(G)}$ ) and the fact that $\delta \mu_{F}^{(G)}$ does not explicitly depend on the system size (cf. eqs. 30, 31): it is directly defined by the stress relaxation function $G(t)$ which is system-size independent for $T \gtrsim T_{g}$ (cf. Fig. 4).

By contrast, at low temperatures $(T \lesssim 0.3)$ the Gaussian deviation is significantly lower than the total $\delta \mu_{F}$ (for both systems). To characterize this discrepancy we introduce a non-Gaussian contribution to the variance of $\mu_{F}$ postulating that

$$
\left(\delta \mu_{F}\right)^{2}=\left(\delta \mu_{F}^{(n G)}\right)^{2}+\left(\delta \mu_{F}^{(G)}\right)^{2}
$$

The non-Gaussian term, $\delta \mu_{F}^{(n G)}$, is plotted against $T$ in Figs. 9c, 11a,b for both systems. It is obviously significant at temperatures well below $T_{g}: \delta \mu_{F}^{(n G)}$ is close to the total $\delta \mu_{F}$ at $T \lesssim 0.3$. On the other hand, $\delta \mu_{F}^{(n G)}$ rapidly decreases near $T_{g}$ and becomes negligible at higher temperatures for both systems. It is furthermore apparent that $\delta \mu_{F}^{(n G)}$ at low $T$ 's is significantly higher for the smaller system (cf. Fig. 9c). 
What is the reason for such system size dependence of the standard deviations $\delta \mu_{F}$ and $\delta \mu_{s f}$ at low $T$ 's (recall that $\delta \mu_{s f} \approx \delta \mu_{F}$, cf. section VI)? Before turning to this question, let us further clarify why the stress fluctuations are nearly Gaussian above $T_{g}$. The general point is that $\sigma(t)$ is never exactly a Gaussian process. However, its statistics are close to Gaussian for large volume $V$ since $\sigma(t)=(1 / V) \int_{V} \sigma(\underline{r}, t) \mathrm{d}^{d} r$ can be considered as a sum of many quasi-independent contributions. Assuming that spatial correlations of $\sigma(\underline{r}, t)$ are short-range, one can easily deduce that the non-Gaussian (nG) correction must scale as the inverse volume: $\operatorname{var}\left(\mu_{F}\right)_{n G} \equiv\left(\delta \mu_{F}^{(n G)}\right)^{2} \propto 1 / V .48$ Above $T_{g}$ this is a small correction, $\operatorname{var}\left(\mu_{F}\right)_{n G} /\left(\delta \mu_{F}\right)^{2} \sim V_{c} / V$, where $V_{c}$ is the stress correlation volume, $V_{c} \ll V$. This estimate has emerged in analogy with the analysis of standard deviations, $\delta \mu_{A}$, of the affine modulus (cf. section V). Below $T_{g}$ the situation is different: here the Gaussian contribution is small as $\delta \mu_{F}^{(G)}$ strongly decreases for long $\Delta t$ at low $T$ (cf. end of section VI). By contrast, the overall $\delta \mu_{F}$ does not vanish (remains finite) for however low $T$ and long $\Delta t$ due to quenched structural correlations (including stress correlations) characterizing the amorphous solid-like state of the system. As a result, for a finite $V$, low $T$ and long $\Delta t$, the variance of $\mu_{F}$ becomes dominated by the volume-dependent non-Gaussian term:

$$
\left(\delta \mu_{F}\right)^{2} \approx \operatorname{var}\left(\mu_{F}\right)_{n G} \propto V_{c} / V \text { at } T \lesssim 0.3
$$

The ideas described above are qualitatively supported with the data shown in Fig. 9c and 11 showing that $\delta \mu_{F}^{(n G)}$ is indeed almost $T$-independent for $T \lesssim 0.3$ (and, besides, it weakly depends on the sampling time $\Delta t$ ). It is remarkable, however, that the system volume dependence of $\delta \mu_{F}^{(n G)}$ is significantly weaker than that implied in eq. 38. Based on our simulation data for the 3 lowest temperatures $(T=0.05,0.10,0.15)$ and different sampling times $\Delta t=10^{5}, 10^{4}$ we find $\operatorname{var}\left(\mu_{F}\right)_{n G} \approx 1.22 \pm 0.1$ for $3072 \times 4$ system and $\operatorname{var}\left(\mu_{F}\right)_{n G} \approx 3.34 \pm 0.3$ for $768 \times 4$ system. These data are compatible with the power law $\operatorname{var}\left(\mu_{F}\right)_{n G} \propto 1 / V^{\alpha}$ with $\alpha \approx 0.7 \pm 0.1$.

As mentioned above, at low temperatures the standard deviation of the terminal modulus $\delta \mu_{s f} \approx \delta \mu_{F}$, and $\delta \mu_{F}$ is dominated by the non-Gaussian contribution, $\delta \mu_{F}^{(n G)}$, so the obtained $V$-dependence is applicable to $\delta \mu_{s f}$ as well: in this regime $\left(\delta \mu_{s f}\right)^{2} \propto 1 / V^{\alpha}$. A similar behavior for the variance of the elastic modulus with $\alpha \approx 0.68 \pm 0.08$ was obtained by studying more system sizes than we do in a simulation study of a 2D binary LJ mixture, ref. 32. (This and a related study ${ }^{35}$ also report an anomalous behavior of nonlinear elastic coefficients for model glass-forming systems at $T<T_{g}$.) The fact that $\alpha<1$ means that the basic physical assumption of just local (short range) structural stress correlations underlying eq. 38 is not valid. We are 
thus driven to conclude that spatial correlations of local stress and of local structure (including local rigidity) in the studied amorphous systems are likely to be long-range (in addition to being persistent in time). Two main possibilities can be anticipated: (i) that the relevant structural correlation length $\xi_{s}$ (characterizing the amorphous inherent structure) is finite, but is larger than (or comparable with) the system size, $\xi_{s} \gtrsim L \sim 20$; (ii) that $\xi_{s}$ is practically infinite and stress correlations follow a power-law decay with the distance $r$. The latter scenario is in harmony with recent theoretical results revealing long-range correlations of the shear stress frozen in the inherent structure showing a universal decay law, $1 / r^{D}$ (here $D$ is the space dimension) ${ }^{13,36,37}$. Interestingly, a large dynamical correlation length is also hinted at by a difference of the long-time behavior of the relaxation modulus $G(t)$ for the two systems (cf. section III). We believe that further studies (perhaps, on larger systems) are required to clarify this issue.

\section{SUMMARY}

1. Analyzing a model LJ oligomer glassformer using MD simulations we established its static and dynamical parameters as a function of temperature and system size (two systems with $3072 \times 4$ and $768 \times 4$ particles have been studied). The shear relaxation modulus $G(t)$, obtained using the fluctuation-dissipation relation, eq. 6, generically shows a fast vibrational relaxation with timescale $\tau_{0}$, the terminal relaxation with much longer time-scale $\tau_{\alpha}$, and (on cooling from liquid into the glass state) also an intermediate transient (slowly decaying) quasi-plateau (cf. Fig. 4). The $G(t)$ relaxation for the smaller system compares well with the results for the larger one (reported in refs. 5 and 6). The main difference is that the $768 \times 4$ system shows somewhat longer $\tau_{\alpha}$ in the low-temperature regime $\left(T \lesssim T_{g}\right)$.

2. The effective shear modulus of the system, $\mu_{s f}$ (obtained using the stress-fluctuation relations, eq. 12, 13: $\mu_{s f}=\mu_{A}-\mu_{F}$, where the fluctuation modulus $\mu_{F}$, eq. 14 , depends on the sampling time $\Delta t$ ) shows a strong increase as the system is cooled below the glass transition temperature $T_{g}$. The step-like function $\mu_{s f}(T)$ is almost independent of the system size, but it gets shifted to lower temperatures as the sampling time $\Delta t$ is increased. The rise of $\mu_{s f}$ near $T_{g}$ is always sharp but continuous. The transition is narrow, its relative width, $\delta T / T_{g} \sim 0.15$, is the same for both systems, and it does not show a visible tendency to decrease as $\Delta t$ gets longer for $\Delta t>10^{4}$ (cf. section IV). ${ }^{49}$ (Here $\delta T=-\mu / \max \left(\partial \mu_{s f} / \partial T\right)$, where $\mu$ is $\mu_{s f}$ right below the transition.) Theoretically, we anticipate a logarithmic increase of the transition steepness at longer 
$\Delta t: T_{g} / \delta T \propto \ln \Delta t$ (as argued in section VII A). Curiously, at low temperatures below the transition zone the shear modulus $\mu_{s f}$ is a bit higher for the smaller system in spite of its slightly lower density.

3. Analyzing the instantaneous affine shear modulus $\mu_{A}$ we revealed that it is dominated by the contribution of bonds connecting the monomers in chains. A moderate increase of $\mu_{A}$ at low $T$ 's is mainly due to an enhancement of LJ interactions as the density gets slightly higher. The affine modulus does not depend on the system size. By contrast, $\operatorname{var}\left(\mu_{A}\right)$ depends on the system volume as $V_{c} / V$, where $V_{c}$ is the effective correlation volume corresponding to just one particle $\left(V_{c} \sim 1\right)$ suggesting that local contributions to the modulus are virtually independent. Noteworthily, the volume $V_{c}$ does not increase as the temperature is lowered (cf. eq. 22).

Moreover, we found that the variance of instantaneous $\mu_{A}, \operatorname{var}\left(\mu_{A}\right)$, is roughly independent of temperature. Therefore the observed strong increase of the variance of the time-averaged $\mu_{A}$ at low $T$ 's is solely due an increase of the relevant relaxation time worsening the statistics of $\mu_{A}$.

Remarkably, we also found that orientational pre-averaging of $\mu_{A}$ (by rotations of the coordinate frame) leads to a drastic improvement of its statistics: the variance of $\mu_{A}$ decreases by a factor between 40 and 600 as a result (with the most dramatic decrease at the lowest temperature). By contrast, averaging over just 3 fixed shear planes (xy, yz, xz) leads to a reduction factor $\sim 3$. The effect of full pre-averaging has a simple meaning: fluctuations of $\mu_{A}$ are generally due to variations of bond orientations and bond length. As shown in section V, the bond orientation fluctuations are dominant, but are completely wiped out by the orientational averaging.

4. To characterize the heterogeneous nature of the glass-forming systems we obtained the standard deviations of $\mu_{s f}$ and $\mu_{F}$ among different independent configurations and found that they are always nearly equal: $\delta \mu_{s f} \approx \delta \mu_{F}$. For both systems these deviations show a pronounced peak near $T_{g}$ in agreement with the results of refs. 5 and 6 for the larger system. For the smaller system the peak gets a bit higher and broader. Its height also slightly increases for longer sampling time $\Delta t$. The peak of $\delta \mu_{s f}$ reflects a sharp transition from liquid-like to solid-like behavior; its emergence is correlated with a strong variation of $\mu_{s f}$ near $T_{g}$ (a high slope $-\partial \mu_{s f} / \partial T$ ).

5. We developed a quantitative theory predicting $\delta \mu_{F}$ in terms of the relaxation modulus $G(t)$. The theory is in excellent agreement with the simulation results in the liquid regime (cf. Figs. $9 \mathrm{~b}, 10 \mathrm{~b})$. In this regime $\delta \mu_{F}$ can be predicted based on the function $\mu_{F}(\Delta t)$ which is directly related to the relaxation modulus (cf. eqs. 19, 21). The peak region is also quantitatively reproduced by the theory (which works better for the larger system). The theoretical approach is based 
on the Gaussian approximation for stress fluctuations, which is asymptotically exact for large systems, $V \rightarrow \infty$ (note, however, that the convergence is not uniform: the finite 'Gaussian' limit is approached for $L \gg \ell(T)$, where the length-scale $\ell(T)$ strongly increases at low $T)$. In this limit it is valid both in the equilibrium (liquid) state and in the glassy state falling out of equilibrium. The theory thus generally proves that fluctuations of $\mu_{F}$ and $\mu_{s f}$ do not vanish for large $V$, rather they tend to a $T$-dependent finite level.

6. The theoretical Gaussian deviations $\delta \mu_{F}^{(G)}$ strongly decrease at low temperatures, in contrast to simulation data pointing to saturation of $\delta \mu_{F}$ at a significant level at low $T$ for the studied systems. Moreover, the low- $T$ plateau of $\delta \mu_{F}$ strongly decreases as the system gets larger. This effect is attributed to a highly heterogeneous amorphous structure of the supercooled glassy liquids leading to markedly non-Gaussian stress fluctuations coupled to the quenched structural disorder. Our analysis shows that the non-Gaussian part of the variance of shear moduli, $\operatorname{var}\left(\mu_{F}\right)_{n G} \approx \operatorname{var}\left(\mu_{s f}\right)_{n G}$, decreases with the system size as $1 / V^{\alpha}$ with $\alpha<1(\alpha \approx 0.7 \pm 0.1)$. This result indicates that local elastic (structural) properties in the studied amorphous systems must show long-range spatial correlations (since a structure with uncorrelated elements would lead to a $1 / V$ dependence of the variance). Such behavior is reminiscent of the so-called Gardner

transition ${ }^{38}$ possibly associated with a diverging length-scale of static heterogeneity below $T_{g}{ }^{39,40}$. A long dynamical length-scale comparable with the system size is also suggested by the revealed size-dependence (at low temperatures) of the terminal decay of the shear relaxation modulus which is slower for the smaller system (cf. section III).

\section{ACKNOWLEDGMENTS}

We acknowledge a partial support from the International Research Training Group (IRTG) 'Soft Matter Science: Concepts for the Design of Functional Materials'. L.Klochko was also supported by the Université de Strasbourg.

\section{REFERENCES}

${ }^{1}$ J.-L. Barrat, J.-N.Roux, J.-P. Hansen, and M.L.Klein, Europhys. Lett. 7, 707 (1988).

${ }^{2}$ A. Zaccone and E. M. Terentjev, Phys. Rev. Lett. 110, 178002 (2013). 
${ }^{3}$ J. P.Wittmer, H. Xu, P. Polinska, F.Weysser, and J. Baschnagel, J. Chem. Phys. 138, 12A533 (2013).

${ }^{4}$ D. Li, H. Xu, and J. P. Wittmer, J. Phys.: Condens. Matter 28, 045101 (2016).

${ }^{5}$ I. Kriuchevskyi, J. P. Wittmer, H. Meyer, and J. Baschnagel, Phys. Rev. Lett. 119, 147802 (2017).

${ }^{6}$ I. Kriuchevskyi, J. P. Wittmer, H. Meyer, O.Benzerara, and J. Baschnagel, Phys. Rev. E 97 , 012502 (2018).

${ }^{7}$ I. Kriuchevskyi, J. Wittmer, O. Benzerara, H. Meyer, and J. Baschnagel, Eur. Phys. J. E 40, 43 (2017).

${ }^{8}$ S. Frey, F. Weysser, H. Meyer, J. Farago, M. Fuchs, and J. Baschnagel, Eur. Phys. J. E 38, 11 (2015).

${ }^{9}$ M. Allen and D. Tildesley, Computer Simulation of Liquids (Oxford University Press, Oxford, 1994).

${ }^{10}$ S. J. Plimpton, J. Comput. Phys. 117, 1 (1995).

${ }^{11}$ H. Xu, J. P. Wittmer, P. Polinska, and J. Baschnagel, Phys. Rev. E 86, 046705 (2012).

${ }^{12}$ R. B. Bird, R. C. Armstrong and O. Hassager, Dynamics of Polymeric Liquids: Fluid Mechanics, Wiley, 1987.

${ }^{13}$ L. Klochko, J. Baschnagel, J. P. Wittmer and A. N. Semenov, Soft Matter, 2018, 14, 6835.

${ }^{14}$ D. Forster, Hydrodynamic Fluctuations, Broken Symmetry, and Correlation Functions, Perseus Books, NY, 1995.

${ }^{15}$ L. D. Landau and E.M. Lifshitz, Statistical Physics, Pergamon, Oxford, 1998.

${ }^{16}$ J.Hansen and I.McDonald, Theory of Simple Liquids, 3rd ed. (Academic, NY, 2006).

${ }^{17}$ J.P.Wittmer, H.Xu, J.Baschnagel, Phys.Rev.E 91, 022107 (2015).

${ }^{18}$ P.G.Debenedetti, F.H.Stillinger, Nature 410 (2001) 259.

${ }^{19}$ H. Yoshino, J.Chem.Phys. 136, 214108 (2012).

${ }^{20}$ M.Vladkov, J.-L.Barrat, Macromol. Theory Simul. 15, 252 (2006).

${ }^{21}$ Alexei E. Likhtman, Sathish K. Sukumaran, and Jorge Ramirez, Macromolecules 40, 6748-6757 (2007).

${ }^{22}$ L.Berthier, G.Biroli, D.Coslovich, W.Kob, C.Toninelli, Phys.Rev.E 86, 031502 (2012).

${ }^{23}$ S.Karmakar, C.Dasgupta, S.Sastry, PNAS 106, 3675 (2009).

${ }^{24}$ A. Cavagna, Phys. Rep., 2009, 476, 51.

${ }^{25}$ W. Götze, Complex Dynamics of Glass-Forming Liquids: A Mode-Coupling Theory (Oxford University Press, Oxford, 2009). 
${ }^{26}$ G. Szamel and E. Flenner, Phys. Rev. Lett. 107, 105505 (2011).

${ }^{27}$ C. L. Klix, F. Ebert, F. Weysser, M. Fuchs, G. Maret, and P. Keim, Phys. Rev. Lett. 109, 178301 (2012).

${ }^{28}$ C. L.Klix,G.Maret, andP.Keim,Phys.Rev.X5, 041033 (2015).

${ }^{29}$ H. Yoshino and F. Zamponi, Phys. Rev. E 90, 022302 (2014).

${ }^{30}$ J. P. Wittmer, I. Kriuchevskyi, A. Cavallo, H. Xu, and J. Baschnagel, Phys. Rev. E 93, 062611 (2016).

${ }^{31}$ J. P. Wittmer, H. Xu, O. Benzerara, and J. Baschnagel, Mol. Phys. 113, 2881 (2015).

${ }^{32}$ I.Procaccia, C.Rainone, C.Shor, M.Singh, Phys.Rev.E 93, 063003 (2016).

${ }^{33}$ D.P.Landau, K.Binder, A Guide to Monte Carlo Simulations in Statistical Physics, Cambridge U. Press, Cambridge, 2000.

${ }^{34}$ E. Lukacs, Characteristic Functions, Griffin, N.Y., 1970.

${ }^{35}$ H.G.E.Hentschel, S.Karmakar, E.Lerner, I.Procaccia, Phys.Rev.E 83, 061101 (2011).

${ }^{36}$ M.Maier, A.Zippelius, M.Fuchs, PRL 119, 265701 (2017).

${ }^{37}$ M.Maier, A.Zippelius, M.Fuchs, J.Chem.Phys. 149, 084502 (2018).

${ }^{38}$ P.Charbonneau, J.Kurchan, G.Parisi, P.Urbani, F.Zamponi, Nat.Comm. 5, 3725 (2014).

${ }^{39}$ P.Charbonneau, Y.Jin, G.Parisi, C.Rainone, B.Seoane, F.Zamponi, Phys.Rev.E 92, 012316 (2015).

${ }^{40}$ L.Berthier, P.Charbonneau, Y.Jin, G.Parisi, B.Seoane, F.Zamponi, PNAS 113, 8397 (2016).

${ }^{41}$ W.Gotze and L.Sjogren, Rep. Prog. Phys. 55241 (1992).

${ }^{42}$ The volume was obtained by the ensemble- and time-averaging over the second half of the tempering run.

${ }^{43}$ This scheme (the shear barostat) implies that the PBC are generally non-rectangular thus allowing for shearing of the simulation cell. The non-physical MC steps are chosen to be very small in order to sufficiently slow-down a backflow (shear reversal) after an imposed shear strain.

${ }^{44}$ The LJ time unit is $\tau_{L J}=d_{m} \sqrt{m_{m} / \varepsilon}$, where $d_{m}$ is monomer diameter (the distance between monomer centers corresponding to the first knot of the original LJ potential), $m_{m}$ is the monomer mass, and $\varepsilon$ is the depth of the LJ potential well.

${ }^{45} \mathrm{An}$ increase of the transition sharpness reported for the larger $3072 \times 4$ system in refs. 5 and 6 concerns short sampling times, $\Delta t \leq 10^{4}$.

${ }^{46}$ The ratio $\delta \mu_{A}(768) / \delta \mu_{A}(3072)$ is close to 1 in the glass transition region, where $\delta \mu_{A}$ strongly depends on $T$. This feature may be due to a small difference in the effective glass transition 
temperatures between the 2 systems. However, it is likely that the $\delta \mu_{A}$ data for the $3072 \times 4$ system $^{5,6}$ are not really reliable in this region.

${ }^{47} \mathrm{~A}$ different conclusion drawn in the previous works ${ }^{5,6}$ was based on an erroneous analysis of the data.

${ }^{48}$ This conclusion is based on the standard behavior of 'finite-size' deviations from the centrallimit theorem for random variables with symmetric distribution. Alternatively, it can be deduced from the cumulant theory ${ }^{34}$ taking into account that $\operatorname{var}\left(\mu_{F}\right)$ involves only second- and 4 th-order moments of $\sigma$ and that $\langle\sigma\rangle=0$.

${ }^{49}$ There might be 2 reasons for such behavior: (i) the statistics somewhat deteriorate in time averages as the sampling time reaches the upper limit $\Delta t=\Delta t_{\max }=10^{5}$; (ii) during a long production run, $\Delta t \sim \Delta t_{\max }$, the system gets slightly more equilibrated, which may lead to a weak drift of its glass transition temperature (in the course of the simulation) resulting in some widening of the transition.

\section{FIGURE CAPTIONS}

FIG. 1. The volume per monomer at $P=0$ vs. $T$ for the two systems: $N_{m}=3072 \times 4$ (black) and $N_{m}=768 \times 4$ (red). Vertical line: $T_{g}=0.38$.

FIG. 2. The radial distribution functions for non-bonded monomers, $g_{n b}(r)$, for two systems, $3072 \times 4$ and $768 \times 4$ at $T=0.43$ (blue and black curves) and at $T=0.25$ (green and red curves). The curves for different systems superimpose perfectly on one another.

FIG. 3. (a) Temperature dependence of the affine modulus $\mu_{A}$ (blue and green curves) and fluctuation modulus $\mu_{F}$ (black and red curves) for $\Delta t=10^{5}$ for the two systems $(3072 \times 4$ and $768 \times 4$, respectively). (b) Temperature dependence of the quasi-static modulus $\mu_{s f}$ for the same $\Delta t$ for two systems, $3072 \times 4$ (black curve), $768 \times 4$ (red curve).

FIG. 4. Comparison of the shear relaxation moduli $G(t)$ vs. $\log (t)$ for the systems $3072 \times 4$ (black curves) and $768 \times 4$ (red curves) at $T=0.40,0.38,0.36,0.30,0.25,0.15$ (from bottom to top). Note that the dilatometric $T_{g}$ is close to 0.38 .

FIG. 5. (a) Temperature dependencies of $\mu_{F}$ for different sampling times $\Delta t=10^{5}, 5 \cdot 10^{4}, 10^{4}$ for the 2 systems: $3072 \times 4$ (black curves from top to bottom) and $768 \times 4$ (red curves). (b) The $T$-dependencies of $\mu_{s f}$ for different sampling times $\Delta t$ for the 2 systems: $768 \times 4$ (3 red curves and 3 magenta curves, from bottom to top for $\Delta t=10^{5}, 5 \cdot 10^{4}, 10^{4}, 5000,1000,500$ ) 
and $3072 \times 4$ (black curves from bottom to top for $\Delta t=10^{5}, 5 \cdot 10^{4}, 10^{4}$ ). (c) Time-temperature superposition for the effective shear modulus for the $768 \times 4$ system: $\mu_{s f}$ is plotted vs. $x=$ $1 / T+(1 / E) \ln \left(\Delta t_{\max } / \Delta t\right)$, where the activation energy $E=18 \mathrm{LJ}$ units and $\Delta t_{\max }=10^{5}$. The curves correspond to different sampling times: $\Delta t=10^{5}$ (solid black), $5 \cdot 10^{4}$ (dashed black), $10^{4}$ (dotted black), 5000 (solid red), 1000 (dashed blue), 500 (dotted green).

FIG. 6. (a) Comparison of the $T$-dependencies of $\delta \mu_{A}$ for the $3072 \times 4$ system (black curve) with $\delta \mu_{A}$ for the smaller system, $768 \times 4$ (blue). In both cases $\delta \mu_{A}$ is based on the time-averaged $\mu_{A}$ obtained by MD simulations for the ensemble of $m=100$ systems and 3 shear planes. (b) The $T$-dependence of the ratio of $\delta \mu_{A}$ for $768 \times 4$ over that for $3072 \times 4$. The ratio is close to 2 for $T<0.35$ (below $T_{g}$ ) indicating that $\delta \mu_{A}$ follows the classical $1 / \sqrt{V}$ law (self-averaging of $\left.\mu_{A}\right)$.

FIG. 7. The affine modulus and its dispersion for the $768 \times 4$ system. (a) Temperature dependencies of the instantaneous affine modulus $\mu_{A}$ (black curve), its bond contribution $\mu_{A b}$ (green curve), the theoretical $\mu_{A b}$ (red curve), the LJ-interaction term $\mu_{A l j}$ (blue curve). Vertical line corresponds to $T_{g}=0.38$. (b) The standard deviations of the instantaneous affine modulus, $\delta_{1} \mu_{A}$ (black curve), and of its parts: $\delta_{1} \mu_{A b}$ due to bonds (green curve), theoretical $\delta_{1} \mu_{A b}$ (red curve), $\delta_{1} \mu_{A l j}$ due to LJ-interactions (blue curve). The bond and LJ contributions to $\mu_{A}$ are generally rather weakly correlated, so the following rule of additive variances works with good accuracy (relative error $\lesssim 1 \%$ ): $\delta_{1} \mu_{A} \approx \sqrt{\delta_{1} \mu_{A b}^{2}+\delta_{1} \mu_{A l j}^{2}}$. (c) The corresponding relative standard deviations of instantaneous moduli: $\delta_{1} \mu_{A} / \mu_{A}$ (black), $\delta_{1} \mu_{A b} / \mu_{A b}$ (green) and $\delta_{1} \mu_{A l j} / \mu_{A l j}$ (blue). (d) Comparison of $T$-dependencies of instant deviations: $\delta_{1} \mu_{A}$ for the total modulus (black curve), its theoretical bond contribution $\delta_{1} \mu_{A b}$ (red curve) and standard deviations of the total time-averaged modulus, $\delta \mu_{A}$ (blue curve). Note that $\delta_{1} \mu_{A} \approx \delta \mu_{A}$ for $T<0.36$. This means that the inherent structure of the system is virtually frozen in this regime: fluctuations of $\mu_{A}$ along the time-trajectory are negligible. (e) The $T$-dependencies for standard deviations of the time-averaged $\mu_{A}: \delta \mu_{A}$ for a fixed shear plane (black curve); $\delta \mu_{A(\text { or })}$ for the orientationaveraged modulus (red curve). Deviations of the instant but orientation-averaged affine modulus and its parts (due to bonds and LJ interactions): total $\delta_{1} \mu_{A(\text { or })}$ (blue curve); bond contribution $\delta_{1} \mu_{A b(\text { or })}$ (magenta); theoretical deviation due to bonds (dashed black); LJ contribution $\delta_{1} \mu_{A l j(\text { or })}$ (green). (f) $T$-dependence of the ratio $\delta \mu_{A} / \delta \mu_{A(\text { or })}$ (black curve); $\delta_{1} \mu_{A} / \delta_{1} \mu_{A(\text { or })}$ (red); $\delta_{1} \mu_{A b} / \delta_{1} \mu_{A b(\text { or })}$ (blue); theory for the latter (green). 
FIG. 8. Temperature dependencies of the affine modulus $\mu_{A}$, its standard deviation $\delta \mu_{A}$, theoretical prediction for its bond part $\mu_{A b}$, and theoretical deviation of the instant bond contribution to the modulus, $\delta_{1} \mu_{A b}$ (respectively, black, blue, red and green curves) for the $3072 \times 4$ system.

FIG. 9. Standard deviations of the fluctuation modulus for $\Delta t=10^{5}$ : (a) $\delta \mu_{F}$ vs. $T$ for $768 \times 4$ (red curve) and $3072 \times 4$ (black curve) systems. Vertical line: reference $T_{g}=0.38$. (b) Temperature dependence of $\delta \mu_{F}$ (red rhombs and black boxes) and its Gaussian part, $\delta \mu_{F}^{(G)}$ (red and black curves) for $768 \times 4$ and $3072 \times 4$ systems, respectively. (c) Non-Gaussian deviation $\delta \mu_{F}^{(n G)}$ vs. $T$ for $768 \times 4$ and $3072 \times 4$ systems (red and black curves, respectively).

FIG. 10. Standard deviations of the fluctuation modulus for the $768 \times 4$ system: (a) $\delta \mu_{F}$ vs. $T$ for different time windows: $\Delta t=10^{5}$ (black), $5 \cdot 10^{4}$ (red), $10^{4}$ (blue curve). (b) $\delta \mu_{F}$ (black boxes, red rhombs and blue crosses) and its Gaussian contribution $\delta \mu_{F}{ }^{(G)}$ (black, red and blue solid curves) for different $\Delta t=10^{5}, 5 \cdot 10^{4}, 10^{4}$.

FIG. 11. The dependence $\delta \mu_{F}^{(n G)}$ vs. $T$ for different sampling times, $\Delta t=10^{5}, 5 \cdot 10^{4}, 10^{4}$ (black, red and blue curves, respectively): (a) for the $3072 \times 4$ system and (b) for the $768 \times 4$ system.

FIG. 12. The dependence of $\mu_{s f}$ (solid curves) and $\delta \mu_{s f}$ (dotted curves) on $T$ for $\Delta t=10^{5}$ for the two systems $\left(768 \times 4\right.$, red and $3072 \times 4$, black). The $\delta \mu_{s f}$ data for the 2 systems $(768 \times 4$ and $3072 x 4$ ) are indicated with '+' and 'x' symbols, respectively.

FIG. 13. The dependence of the ratio $\delta \mu_{1} / \mu_{1}$ for $768 \times 4$ system (black curve), and the prediction, eq. 36 (red line). 


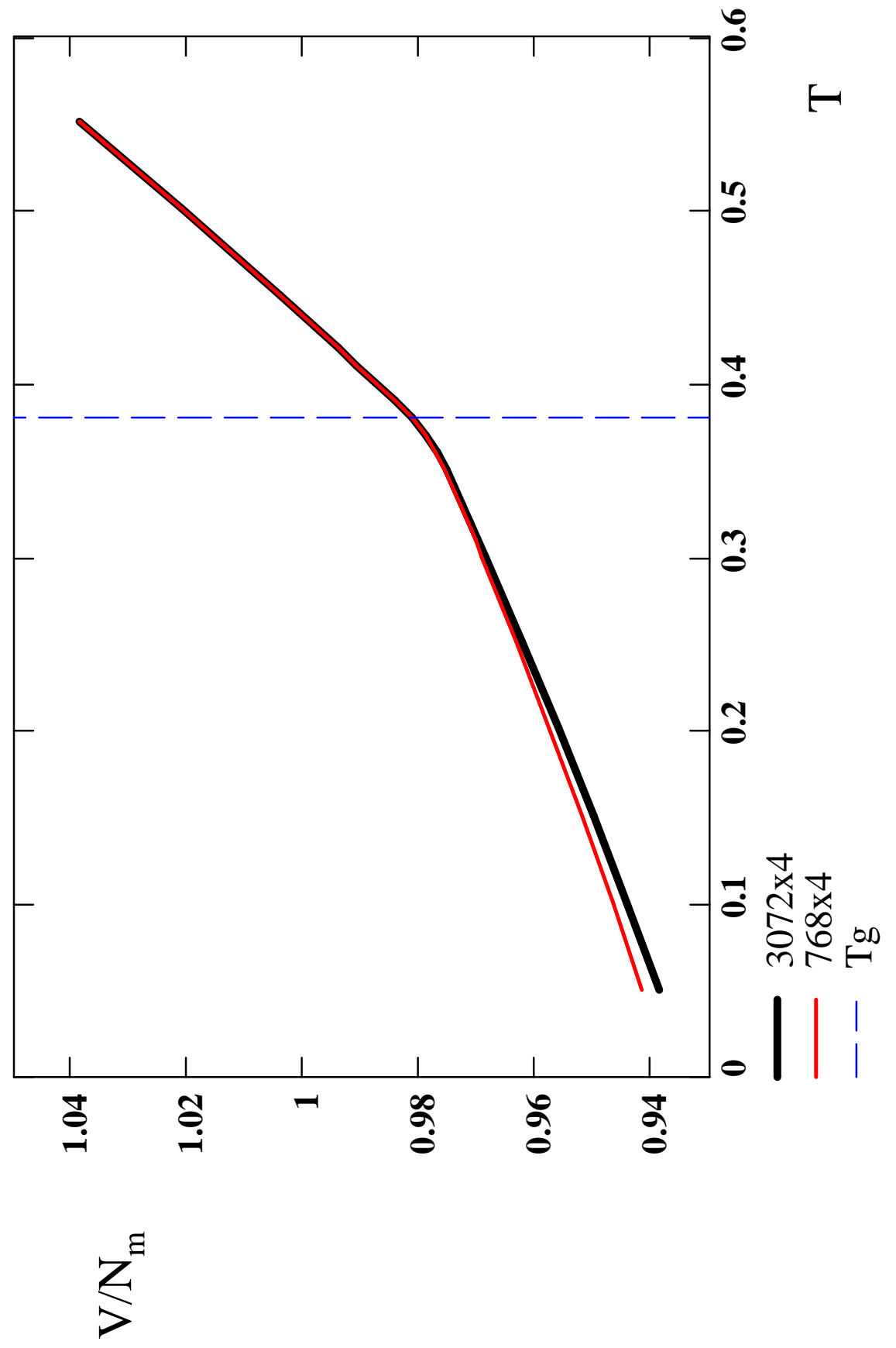

$\underset{\dot{0}}{\dot{0}}$ 


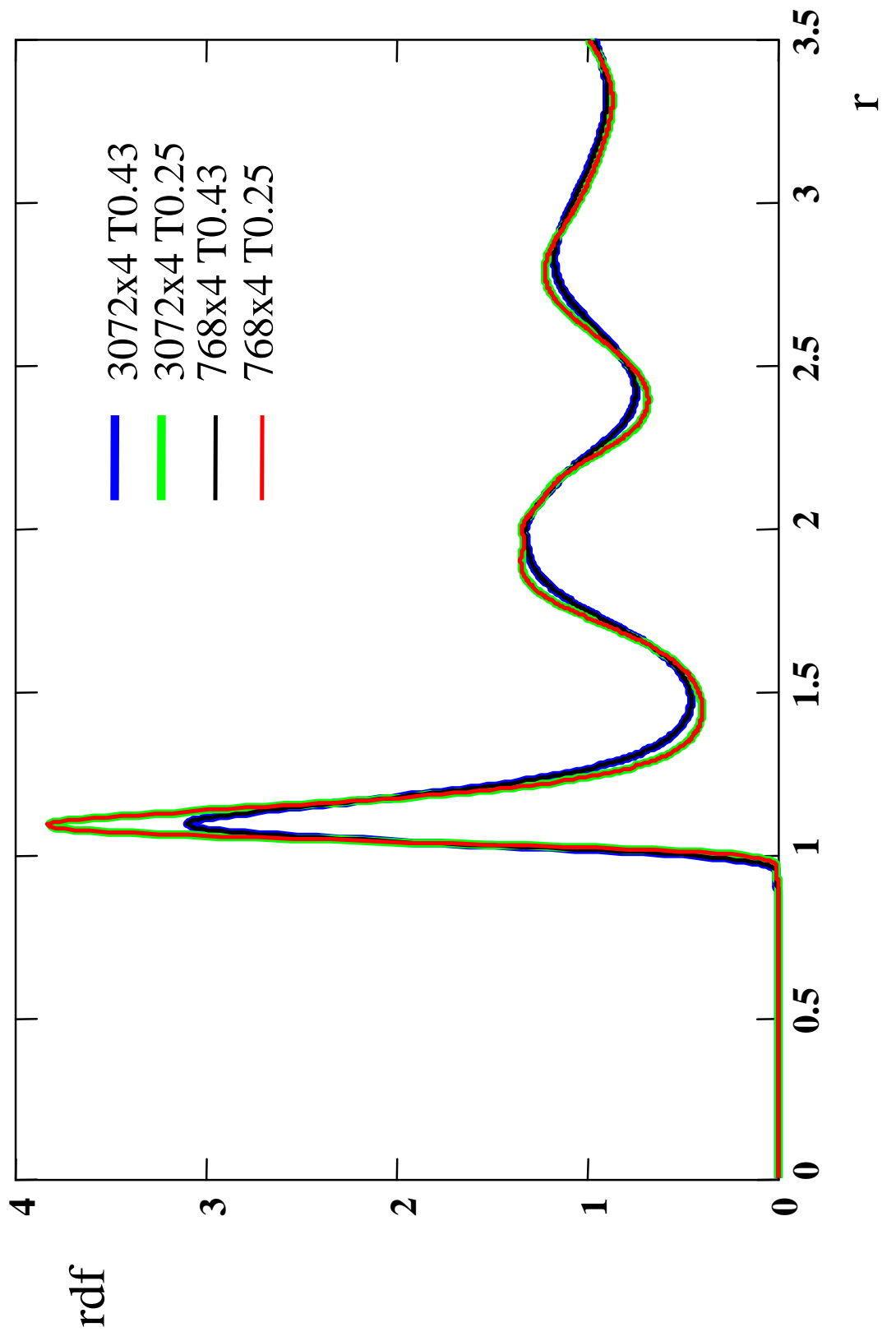

i) 
$\dot{\sigma}$
$\dot{\omega}$
$\dot{\omega}$

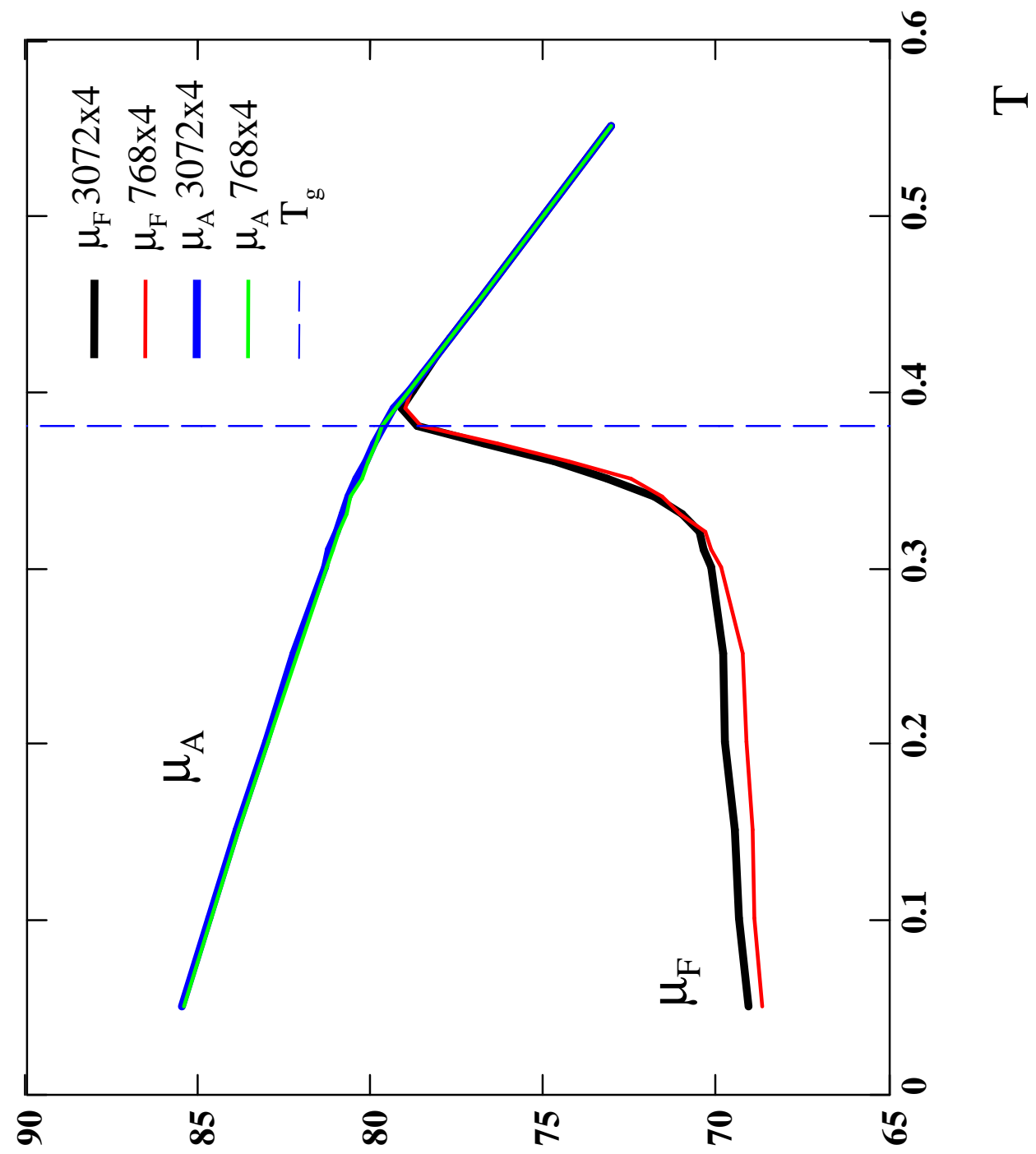




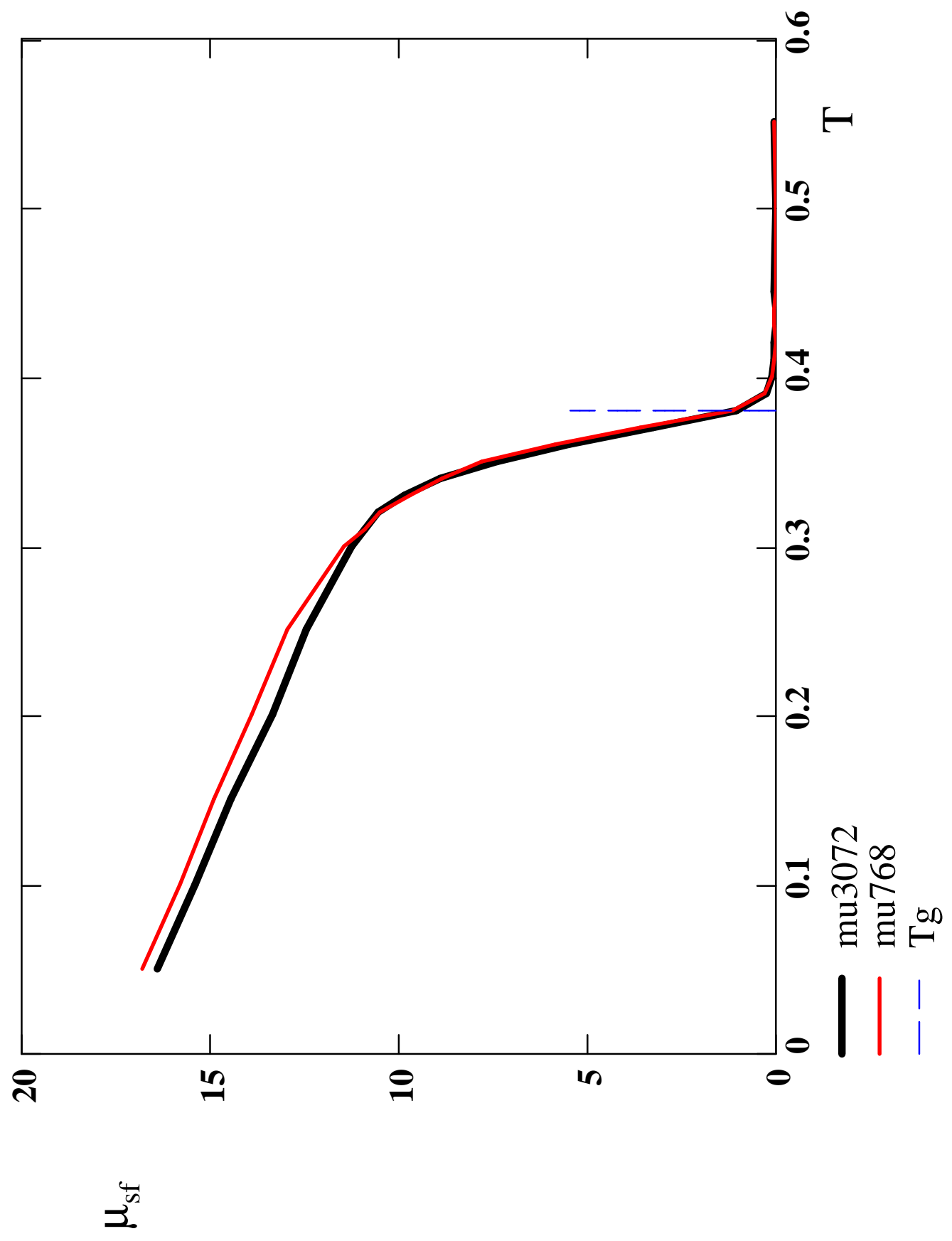

m 定 
$\dot{\forall}$
$\dot{0}$
$\dot{1}$

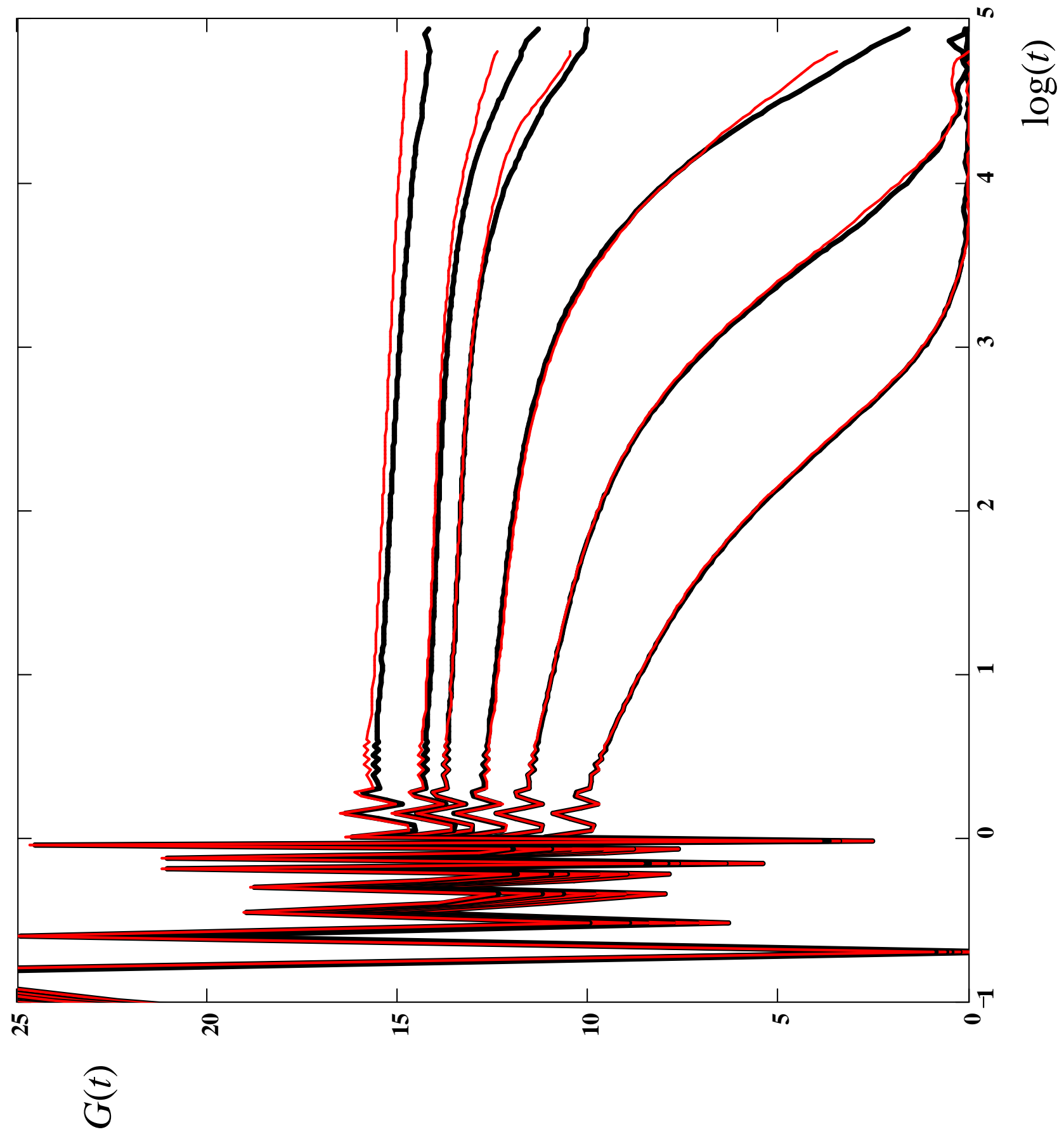




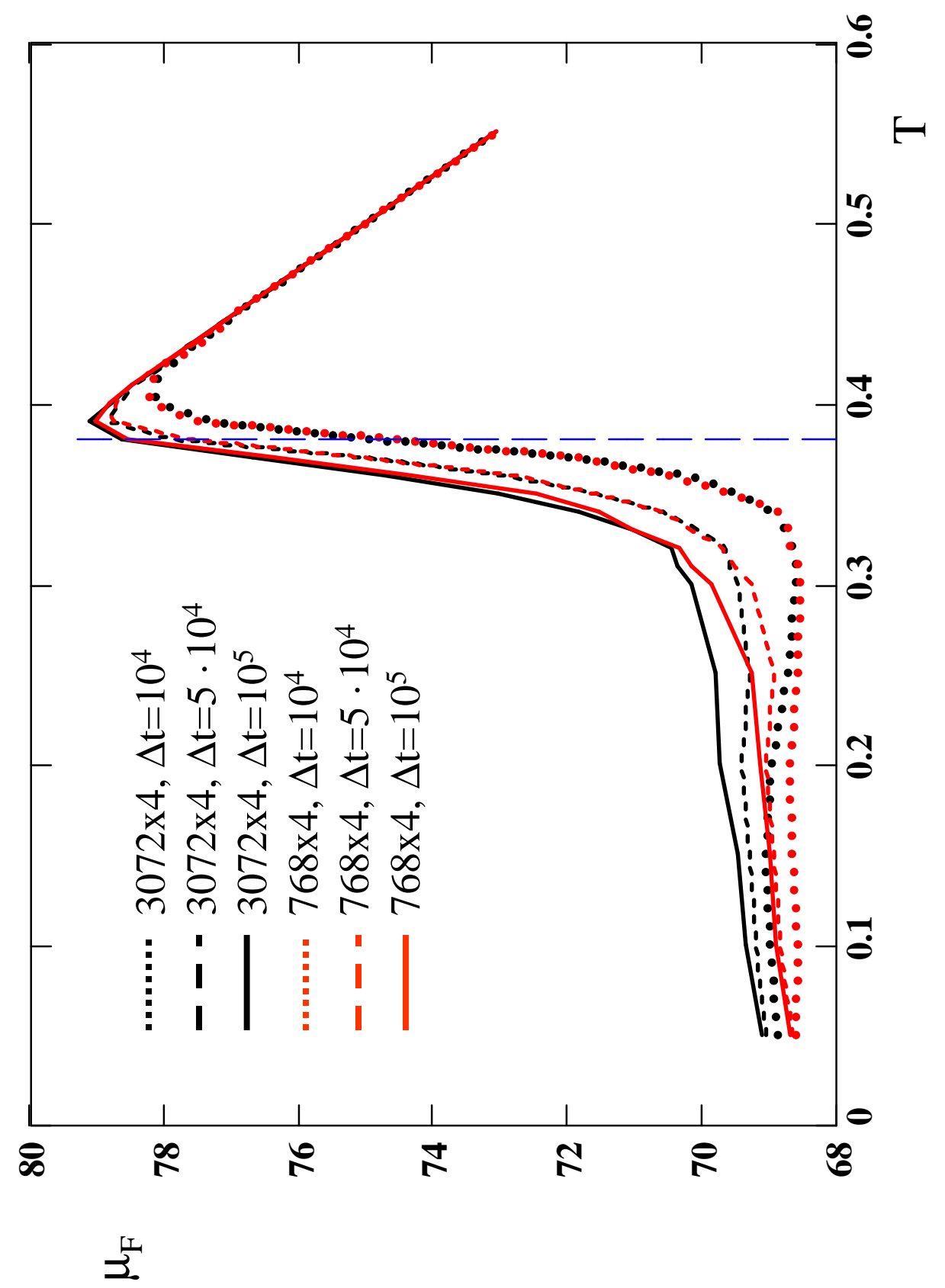

กั के 


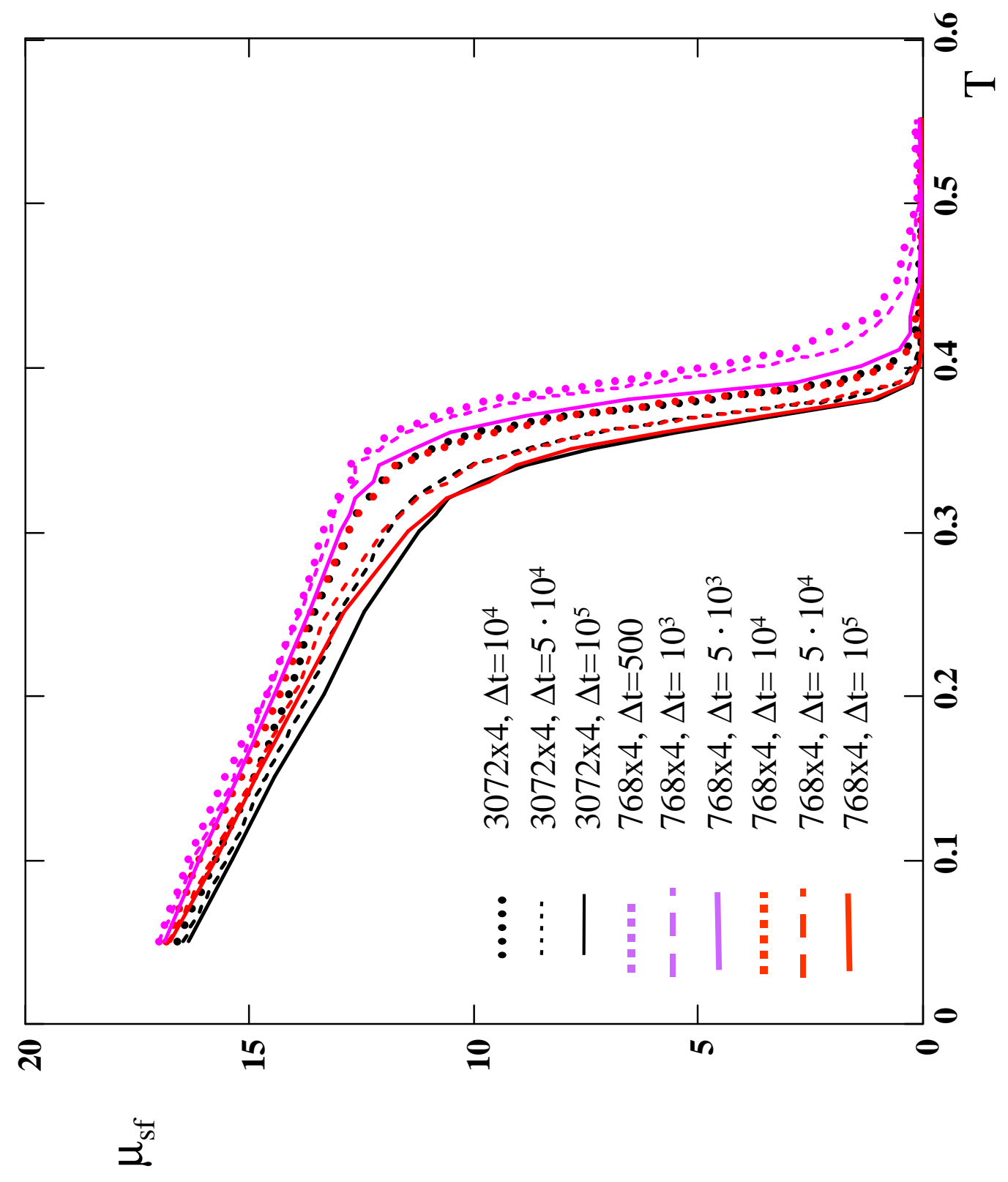

$\dot{0}$
$\dot{0} \dot{1}$ 


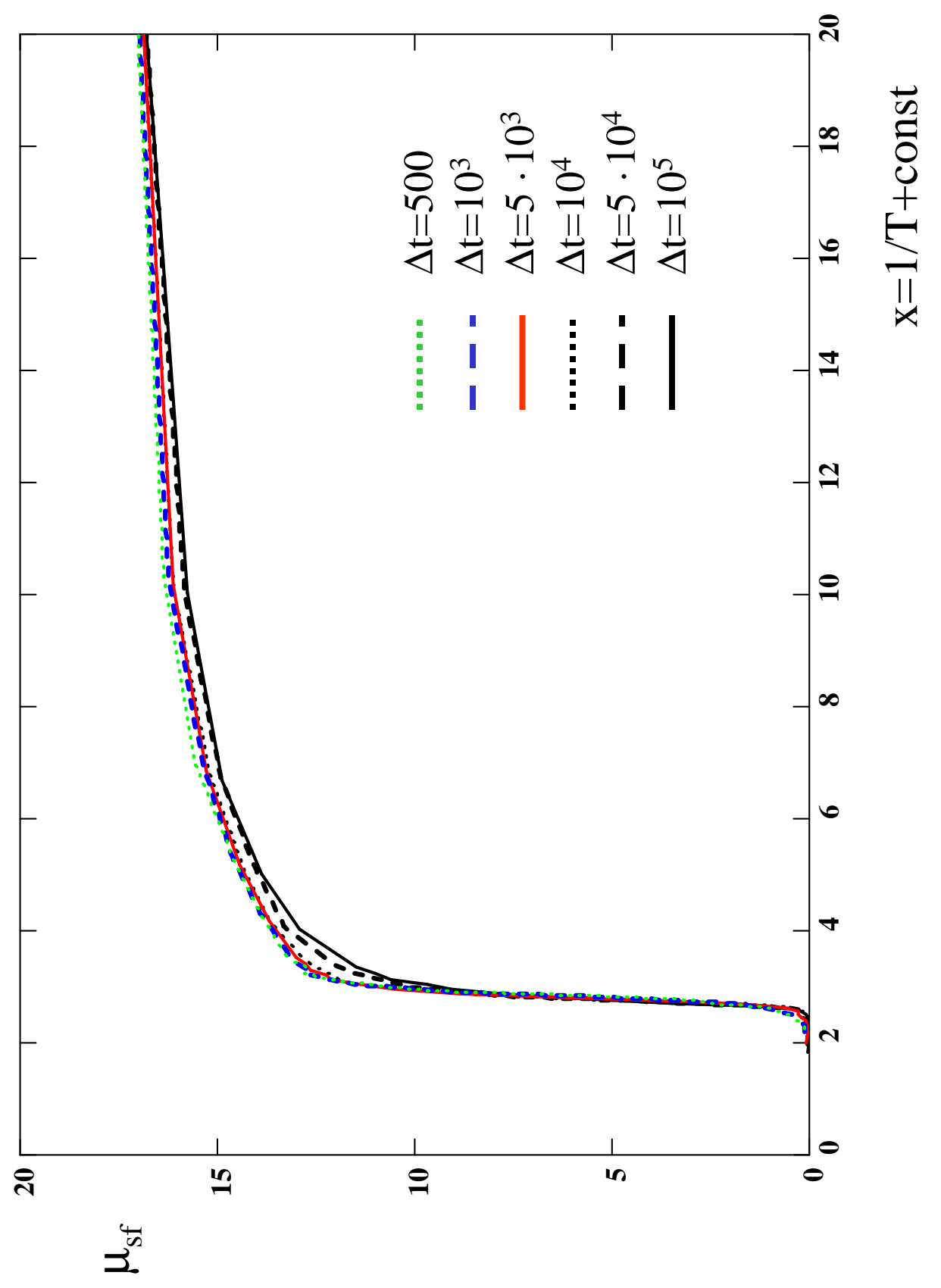

i 

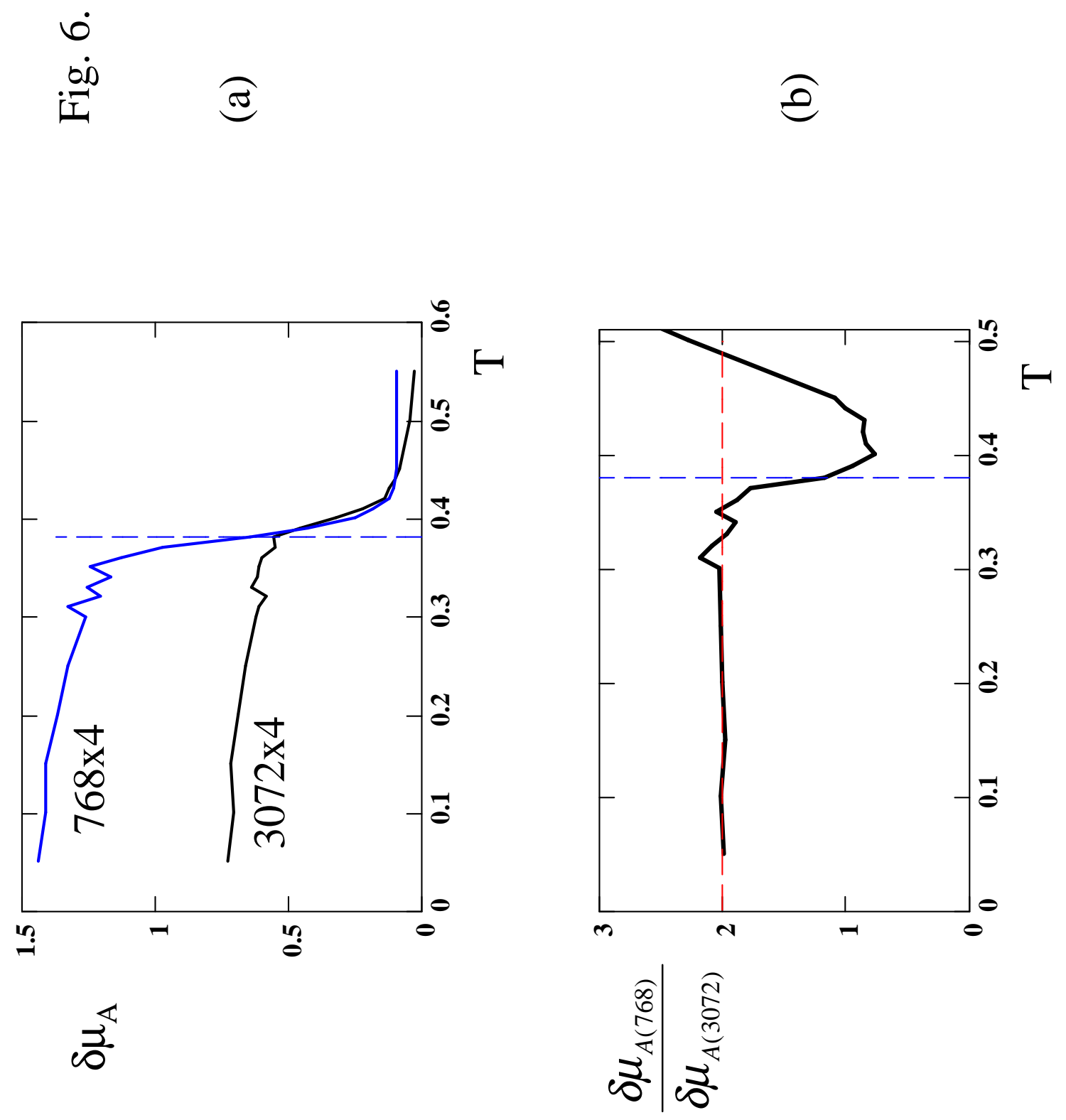


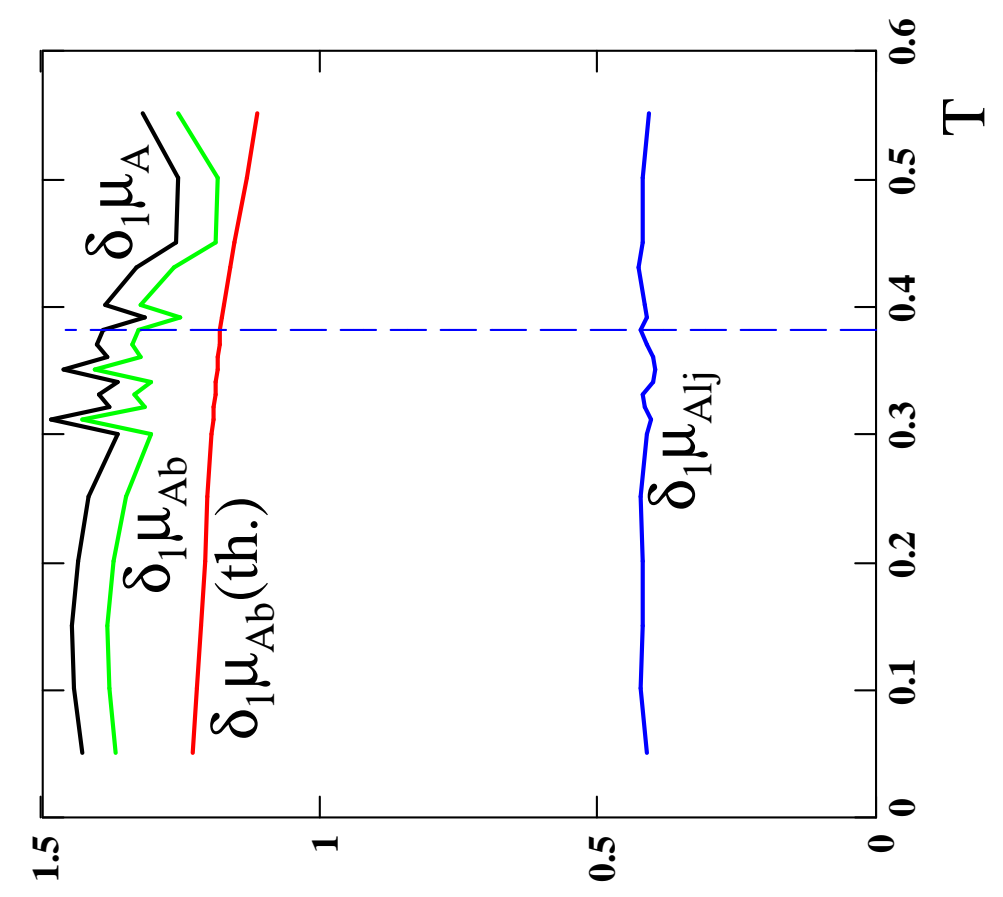

$\hat{e}$

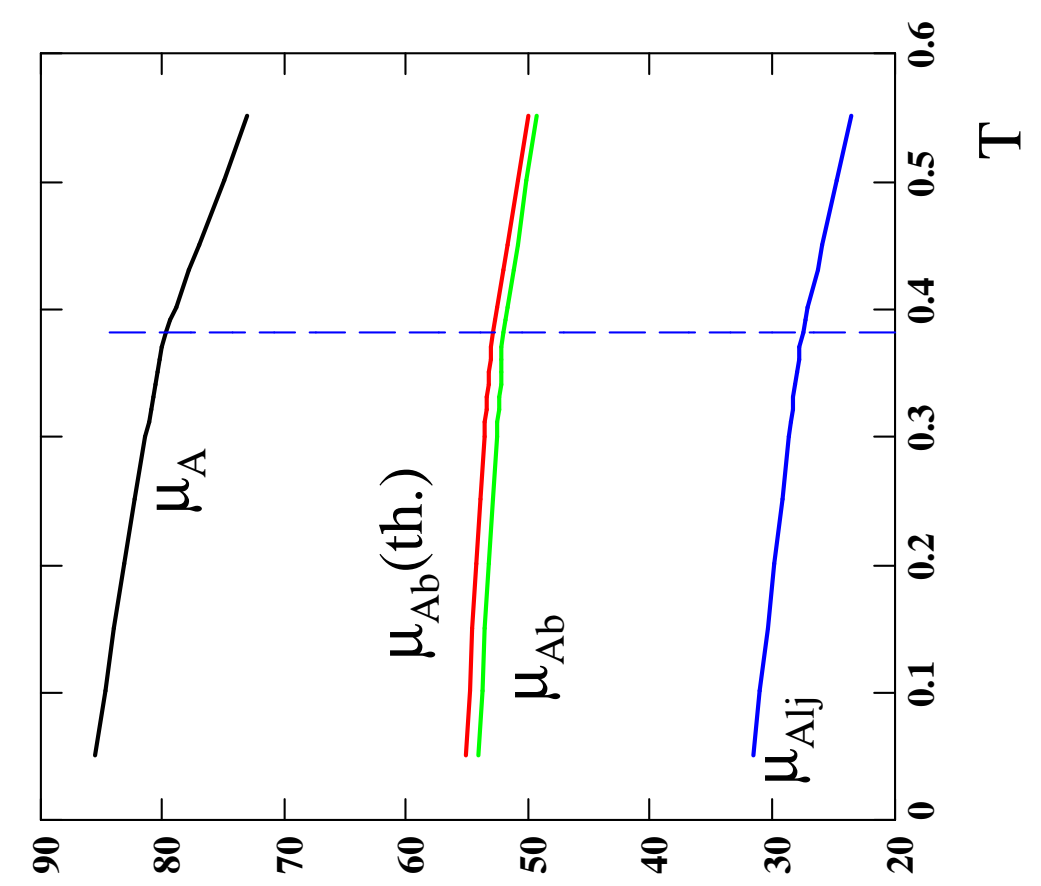

శี

$\dot{i}$ 


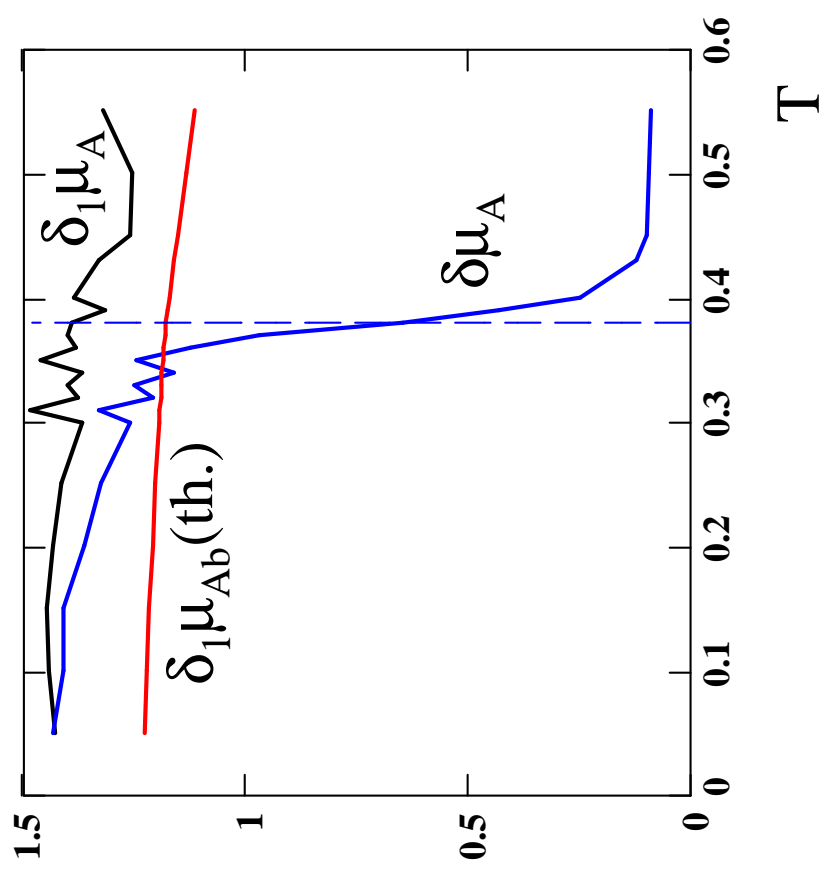

อ

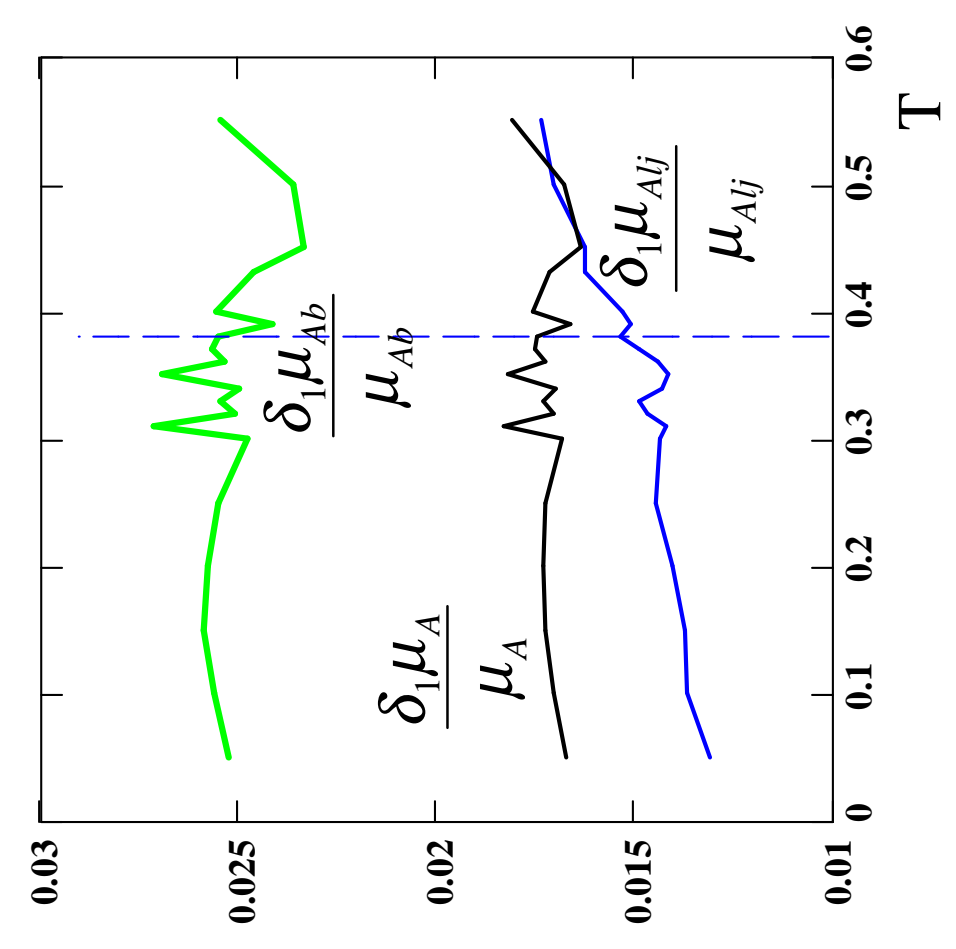

e

$\stackrel{\dot{0}}{\dot{D}}$ 
$\stackrel{\dot{0}}{\dot{0}} \underset{\Theta}{\Theta}$

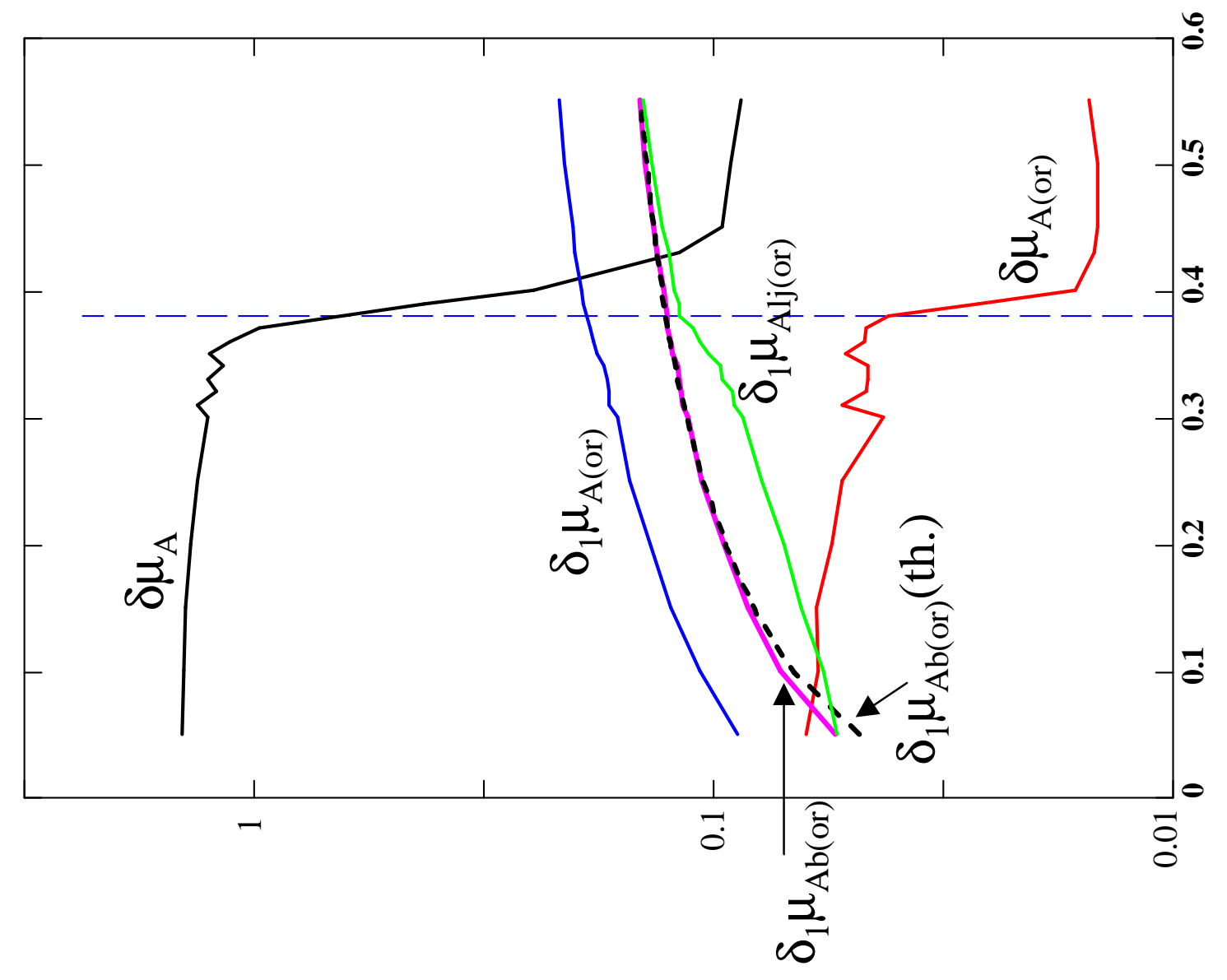


N

定 $\subseteq$

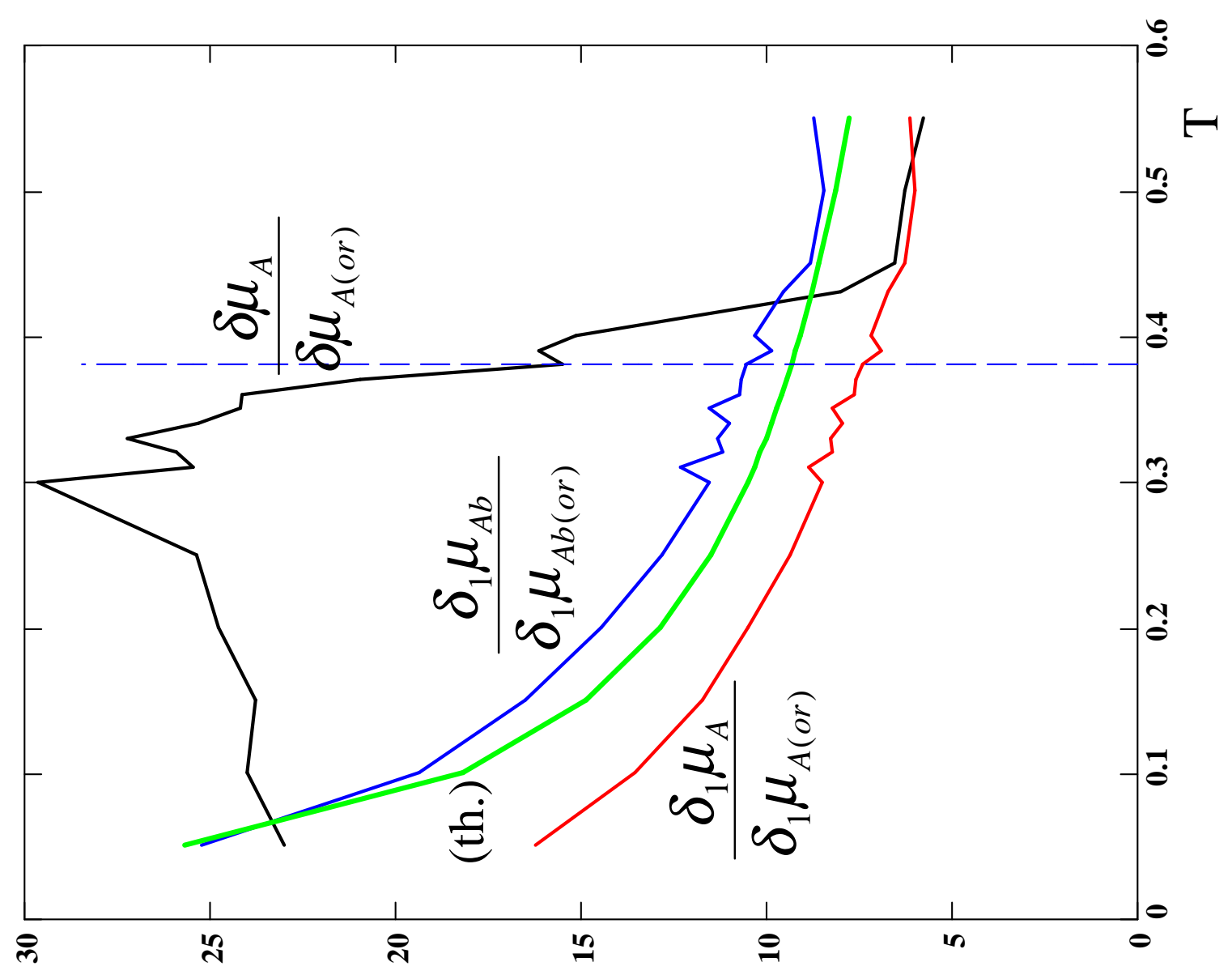


$\infty$

is

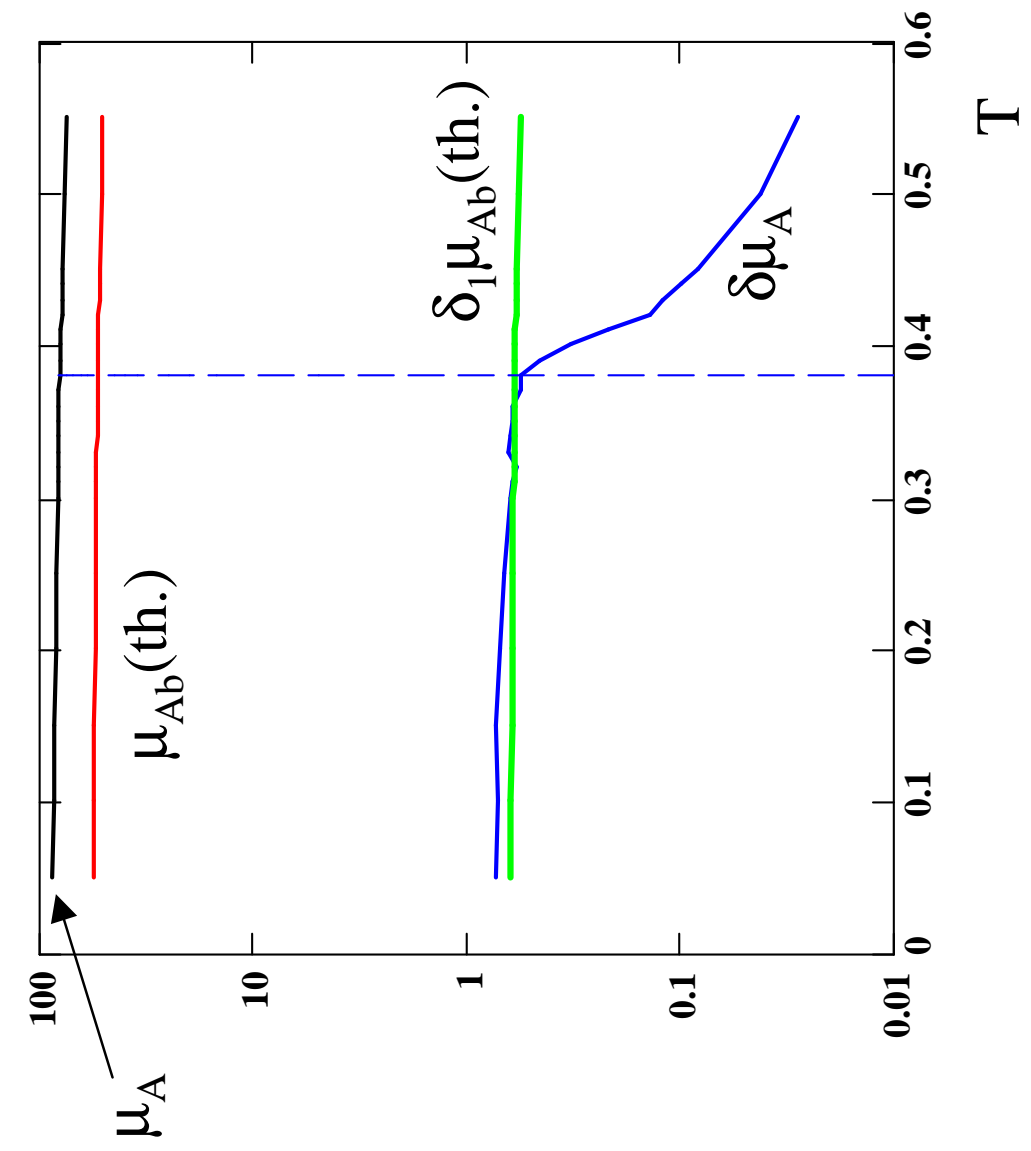




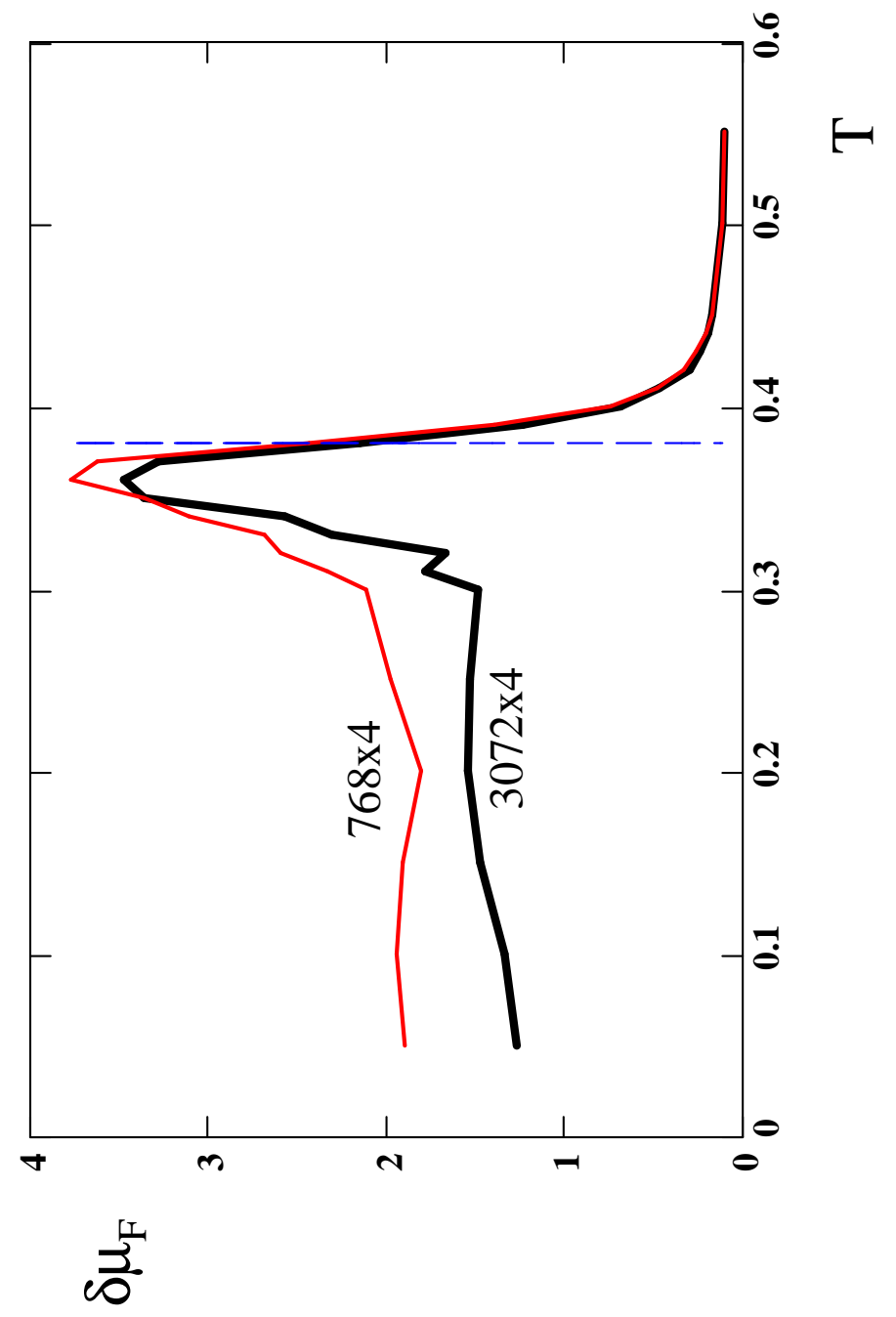

مَ 


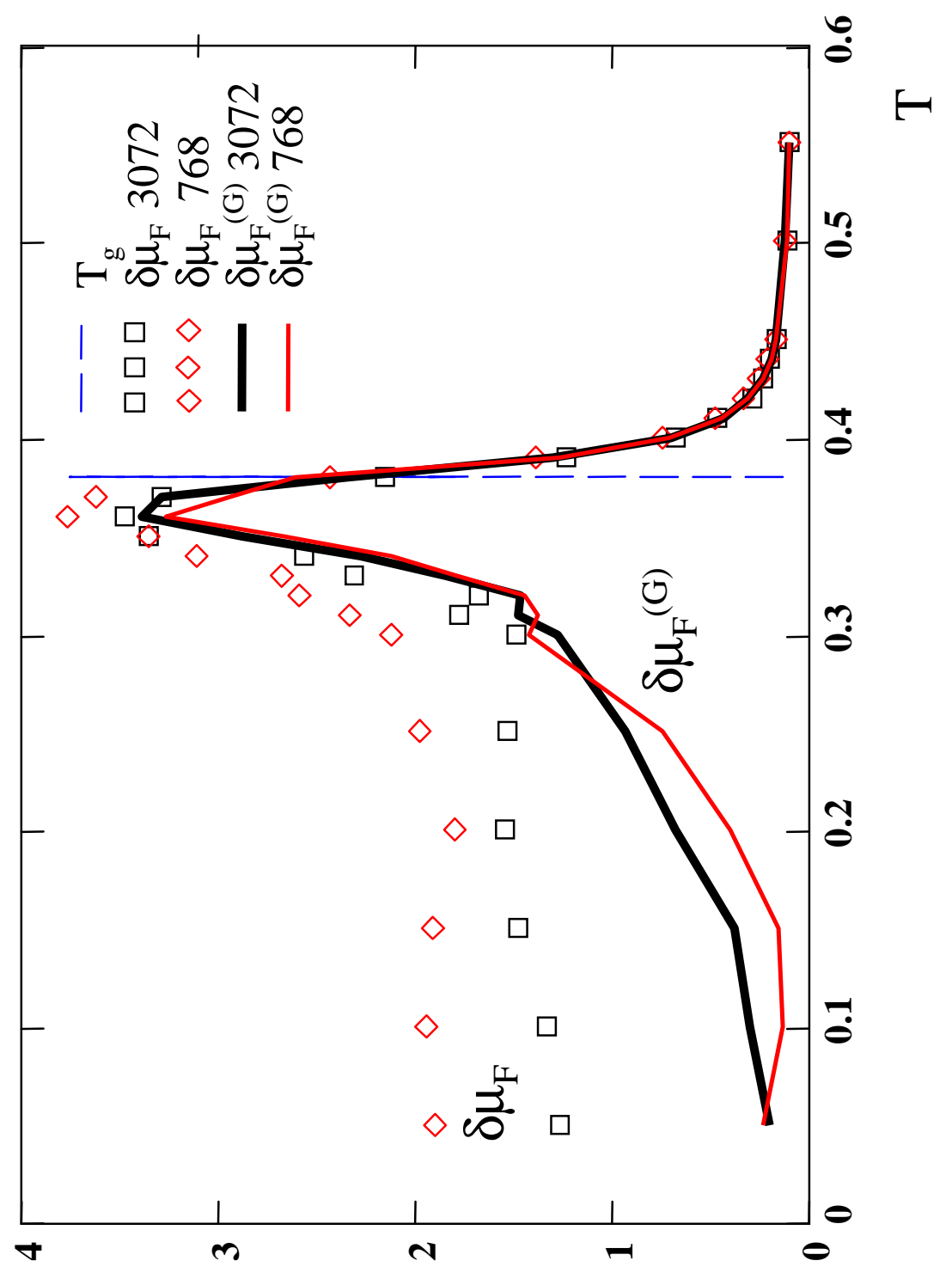

a is 


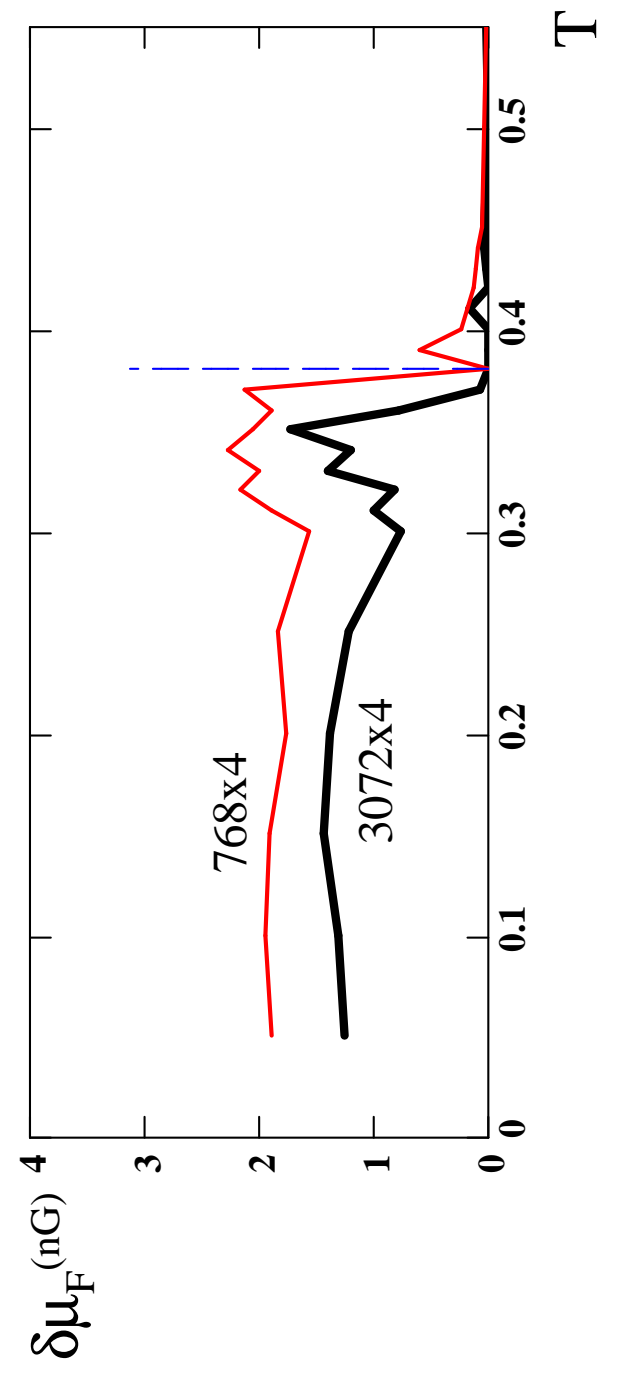

o

is 


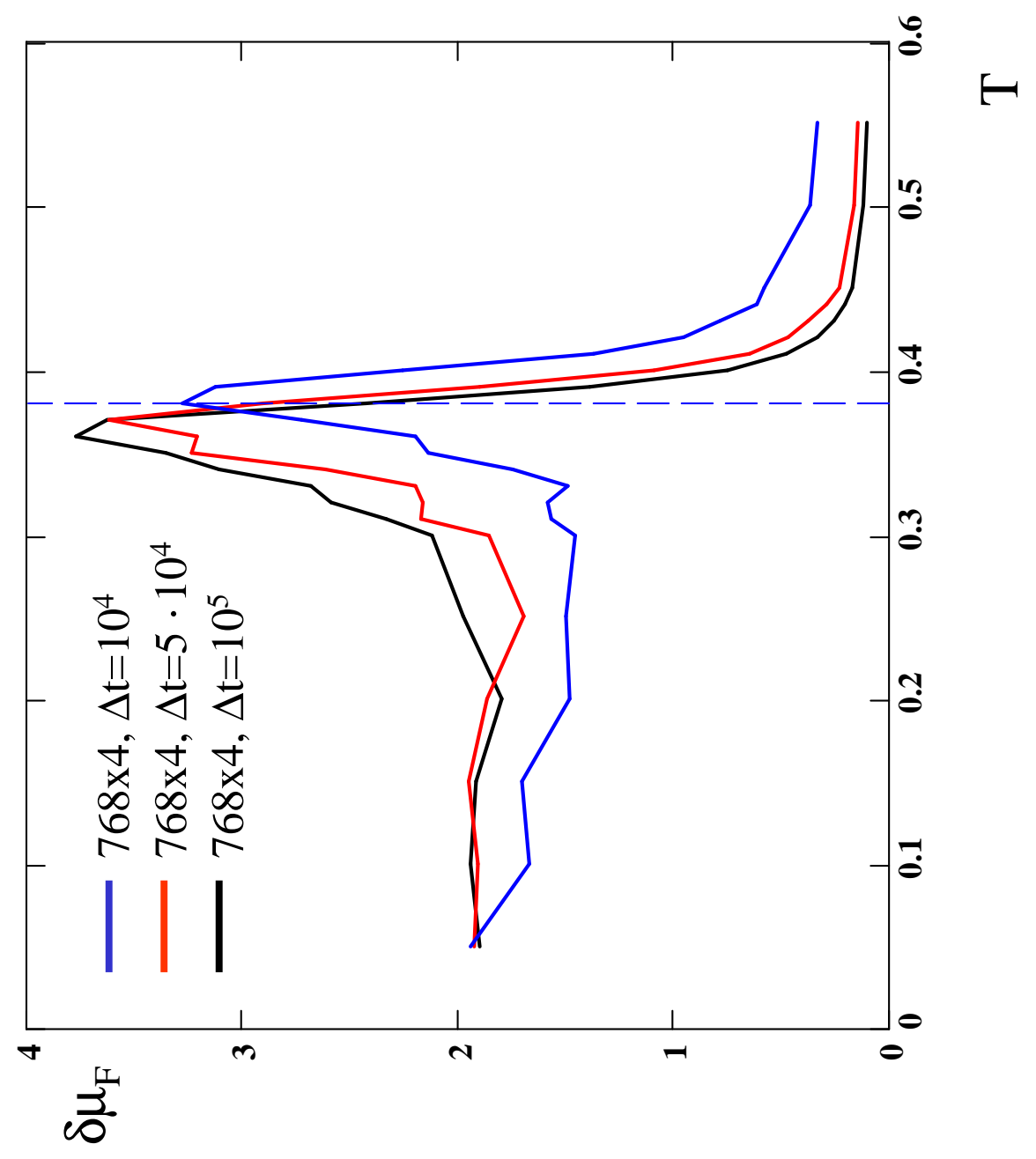

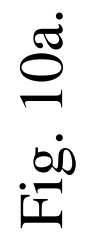




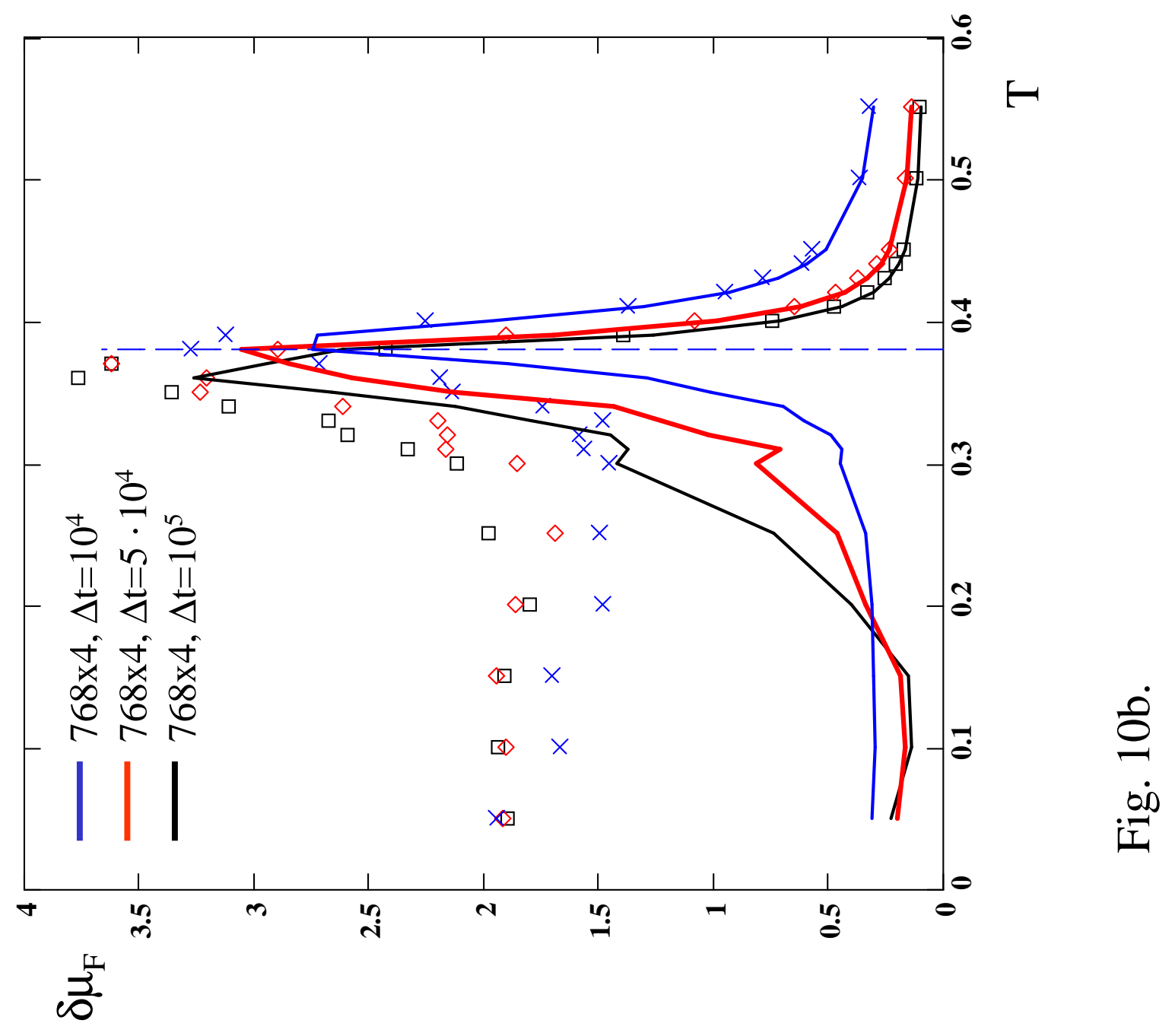




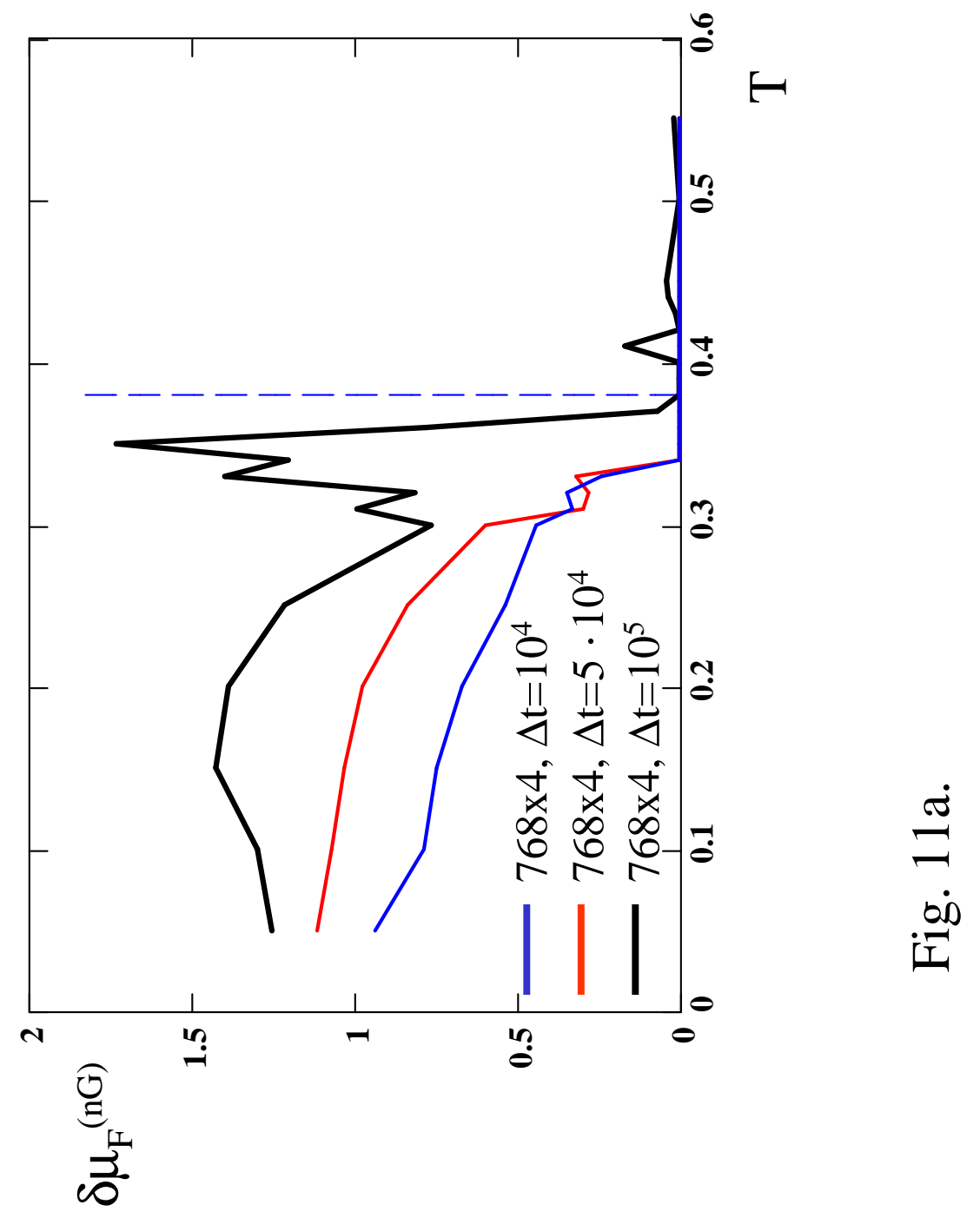




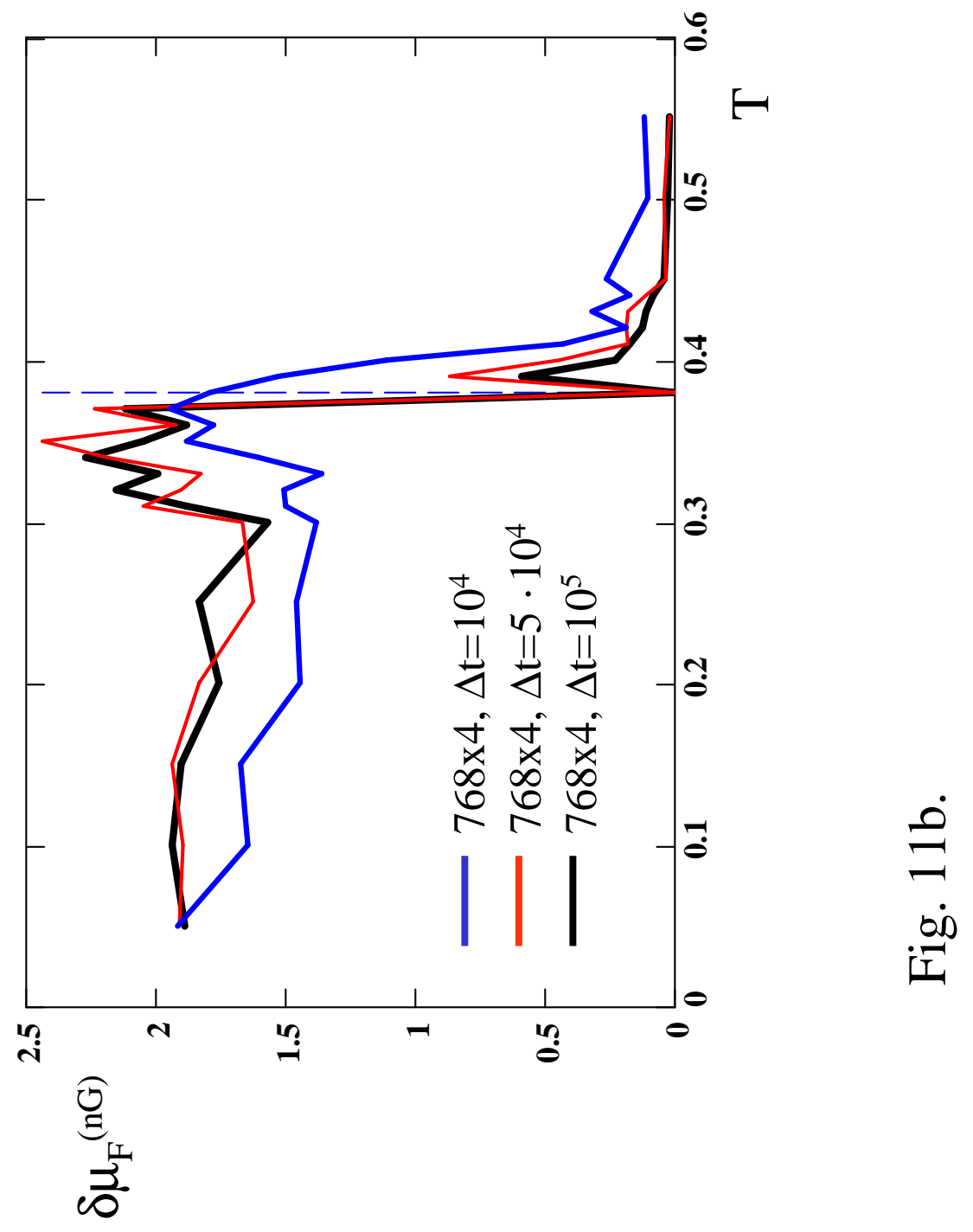




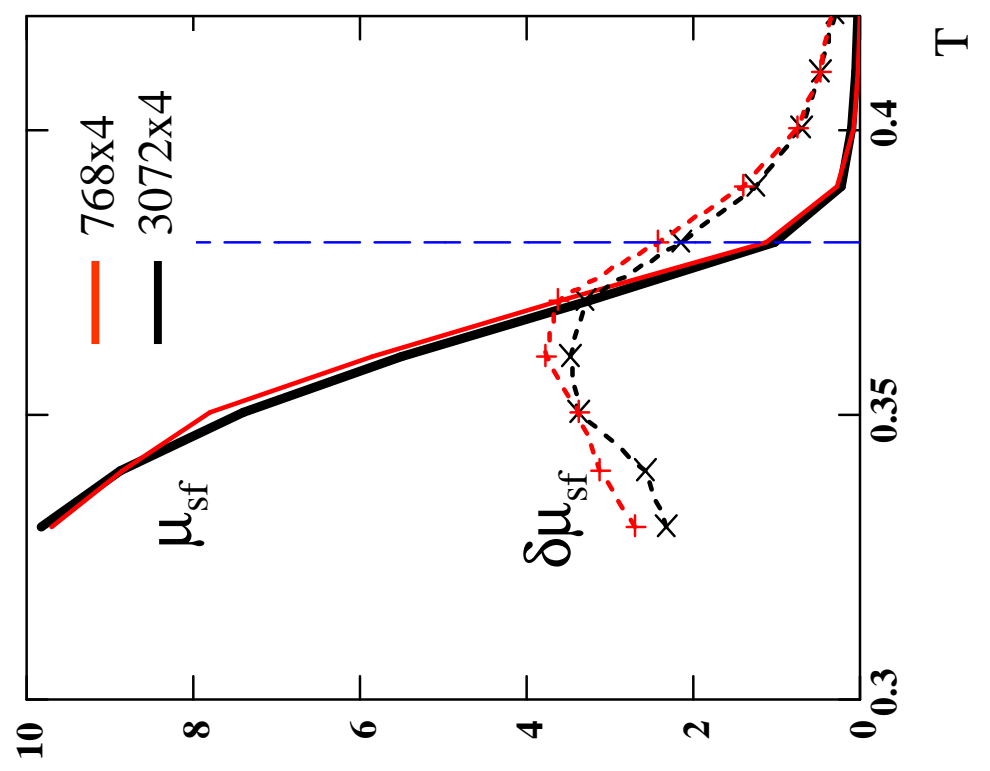

$\underset{\dot{0}}{\dot{0}}$ 


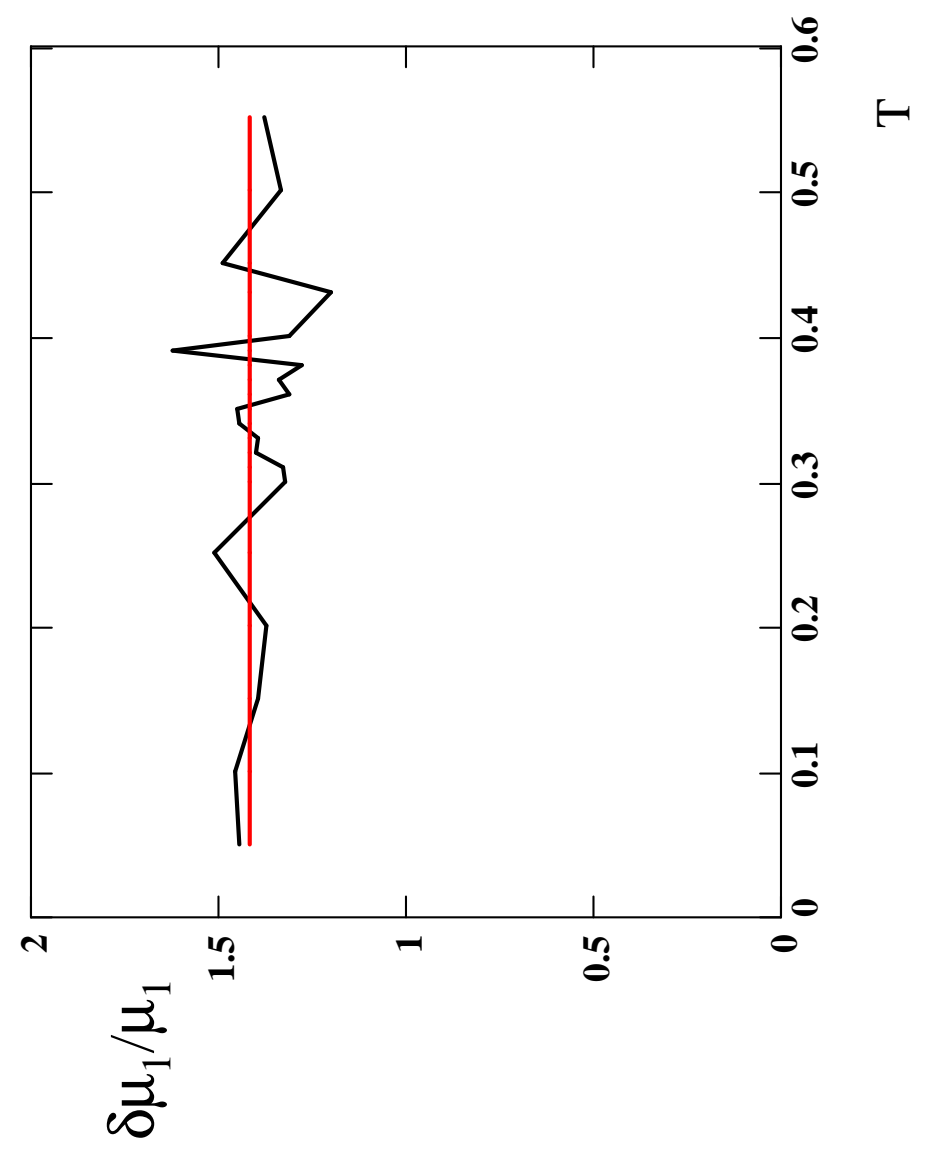

$\underset{\dot{m}}{\dot{m}}$ 